\title{
Reward, Punishment, and Cooperation: A Meta-Analysis
}

\author{
Daniel Balliet \\ Singapore Management University and \\ VU University Amsterdam
}

\author{
Laetitia B. Mulder \\ University of Groningen
}

\author{
Paul A. M. Van Lange \\ VU University Amsterdam
}

\begin{abstract}
How effective are rewards (for cooperation) and punishment (for noncooperation) as tools to promote cooperation in social dilemmas or situations when immediate self-interest and longer term collective interest conflict? What variables can promote the impact of these incentives? Although such questions have been examined, social and behavioral scientists provide different answers. To date, there is no theoretical and/or quantitative review of rewards and punishments as incentives for cooperation in social dilemmas. Using a novel interdependence-theoretic framework, we propose that rewards and punishments should both promote cooperation, and we identify 2 variables - cost of incentives and source of incentives - that are predicted to magnify the effectiveness of these incentives in promoting cooperation. A meta-analysis involving 187 effect sizes revealed that rewards and punishments exhibited a statistically equivalent positive effect on cooperation $(d=0.51$ and 0.70 , respectively). The effectiveness of incentives was stronger when the incentives were costly to administer, compared to free. Centralization of incentives did not moderate the effect size. Punishments were also more effective during iterated dilemmas when participants continued to interact in the same group, compared to both (a) iterated dilemmas with reassignment to a new group after each trial and (b) one-shot dilemmas. We also examine several other potential moderators, such as iterations, partner matching, group size, country, and participant payment. We discuss broad conclusions, consider implications for theory, and suggest directions for future research on rewards and punishment in social dilemmas.
\end{abstract}

Keywords: punishment, reward, cooperation, social dilemma, meta-analysis

\begin{abstract}
Good and evil, reward and punishment, are the only motives to a rational creature: these are the spur and reins whereby all mankind are set on work, and guided.
\end{abstract}

—John Locke, Some Thoughts Concerning Education

If people are good only because they fear punishment, and hope for reward, then we are a sorry lot indeed.

-Albert Einstein, quoted in All the Questions You Ever Wanted to Ask American Atheists

Reward and punishment are incentives that tend to capture strong views of human nature, as well as beliefs regarding public policy, political structures, and organizational systems. Some, like John Locke, believe that incentives are effective tools that help regulate

This article was published Online First May 16, 2011.

Daniel Balliet, School of Social Sciences, Singapore Management University, Singapore, and Department of Social and Organizational Psychology, VU University Amsterdam, Amsterdam, the Netherlands; Laetitia B. Mulder, Department of Human Resource Management and Organizational Behavior, University of Groningen, Groningen, the Netherlands; Paul A. M. Van Lange, Department of Social and Organizational Psychology, VU University Amsterdam, Amsterdam, the Netherlands.

This research was supported by Singapore Management University Faculty Research Grant 09-C242-SMU-009.

Correspondence concerning this article should be addressed to Daniel Balliet, Department of Social and Organizational Psychology, VU University, Van der Boechorststraat 1, 1081 BT, Amsterdam, the Netherlands. E-mail: dp.balliet@psy.vu.nl individuals in their pursuit of self-interest. Others, like Albert Einstein, believe that incentives may undermine autonomy, authenticity, and, most importantly, the true motive to be good. One of the strongest views was expressed by Thomas Hobbes (1651), who argued that people who want collective interest to triumph over selfinterest should support Leviathan, an authority or government that enforces social order. More recently, Hardin (1968) noted that coercion may be the most effective tool to encourage people to sacrifice self-interest for collective benefit. These ideas ignited interdisciplinary research across the biological and social sciences on the effect of incentives as a solution to cooperation (Edney \& Harper, 1978; Fehr \& Gächter, 2000; Lynn \& Oldenquist, 1986; Ostrom, Walker, \& Gardner, 1992; Sigmund, 2007; Yamagishi, 1986). This research has generally supported the position of Hobbes and Hardin: Incentives for cooperation do encourage people to sacrifice their self-interest for collective benefit (e.g., Gächter, Renner, \& Sefton, 2008; Rand, Dreber, Ellingsen, Fudenberg, \& Nowak, 2009).

Aligned with Albert Einstein's view, however, other perspectives suggest that incentives may undermine cooperation. ${ }^{1}$ For

\footnotetext{
${ }^{1}$ In this article, we use the term incentives to refer to both rewards and punishments of behavior in social dilemmas. Moreover, we use the term effectiveness of incentives to refer to the positive effect of incentives on cooperation in social dilemmas. We are not referring to the overall efficiency of incentives as a solution to social dilemmas. Although incentives may be effective at enhancing cooperation in social dilemmas, incentives could still remain a very inefficient solution to a social dilemma due to the cost of monitoring behavior and providing the incentive.
} 
example, incentives may undermine autonomy and the intrinsic motivation to cooperate (Deci \& Ryan, 2000; Ryan \& Deci, 2000), which can reduce persistence in cooperation (Cardenas, Stranlund, \& Willis, 2002; Lepper \& Greene, 1978), result in resistance to comply with the external forces (Brehm, 1966), and/or influence a decline in cooperation if the incentive is no longer present (Deci, Koestner, \& Ryan, 1999). Social incentives can also evoke costly retaliation (DenantBoemont, Masclet, \& Noussair, 2007; Hopfensitz \& Rueben, 2009; Oliver, 1980), transform an ethical decision into a business decision (Gneezy \& Rustichini, 2000; Tenbrunsel \& Messick, 1999), and even undermine trust in others (Chen, Pillutla, \& Yao, 2009; Mulder, van Dijk, De Cremer, \& Wilke, 2006a), and thus create their own necessity (Mulder, van Dijk, Wilke, \& De Cremer, 2005). Although these disadvantages of incentives may not be a threat to their effectiveness as long as they are strong and inescapable (Tenbrunsel \& Messick, 1999), it is often unfeasible and/or undesirable to install strong incentives for cooperation and a watertight monitoring system. Whereas incentives can exert negative psychological effects (e.g., reactance, a lack of intrinsic obligation and distrust), one cannot expect an incentive system to be an all-encompassing and long-term solution to social dilemmas. Instead, one should understand under what conditions incentives are most effective.

The major purpose of the present meta-analytic review is to provide a comprehensive analysis of three broad questions. First, do incentives to cooperate promote and sustain cooperation in small group social dilemmas? Second, what variables might influence the effectiveness of incentives? Finally, do reward and punishment differ in their ability to promote and sustain cooperation? As we discuss shortly, we adopt an interdependence-theoretical analysis for understanding whether incentives might promote cooperation and when these incentives might be especially effective.

\section{Social Dilemmas and the Free-Rider Problem}

Social dilemmas are situations of interdependence characterized by a conflict between immediate self-interest and longer term collective interest (Dawes, 1980; Van Lange \& Joireman, 2008). Many social dilemmas take the form of either a public goods dilemma or a resource dilemma. In a public goods dilemma, people decide how much to contribute to the installment, maintenance, or improvement of a public good, such as paying for public transportation, contributing to a group project, or engaging in teamwork (Gächter \& Herrmann, 2009). Similarly, in a resource dilemma, people decide how much to take from a shared resource. For example, fishermen may decide how many fish to catch in an area where certain fish are endangered, knowing that, over time, overharvesting will lead to depletion of that particular fish (Komorita \& Parks, 1995).

One major challenge is the free-rider problem. In social dilemmas, it is individually tempting to contribute as little as possible to public goods or take as much as possible in resource dilemmas, while enjoying the benefits of others' cooperation, such as the provision of a public good or access to a sustainable resource. To reduce free riding and encourage cooperation, authorities often provide rewards (e.g., public recognition) and/or punishments (e.g., fines). Additionally, groups and collectives often develop social norms that are informally enforced to regulate individual behavior and promote cooperation. How can one best understand the effect of these incentives on cooperation in social dilemmas?

\section{An Interdependence Analysis of Rewards and Punishment in Social Dilemmas}

Since Skinner (1953), research has shown that reinforcements and punishments are important forms of situational feedback that promote learning and performance (for an overview, see Kazdin, 2001). Performance and learning are outcomes that are relevant to the individual, and they typically serve one's own self-interest in the shorter term, in the case of performance (e.g., receiving a high grade), or longer term, in the case of learning (e.g., mastering a particular skill). In contrast, incentives in the context of social dilemmas are conceptually distinct, in that they serve as motivators of cooperation and include both (a) a cost to oneself and (b) a benefit to all other members of the group. Moreover, the benefit for others, in absolute terms, is greater than the cost to oneself. Thus, in social dilemmas, incentives seek to motivate people to act against their immediate self-interest so as to serve the collective interest.

Classic and contemporary formulations of interdependence theory provide a conceptual framework that acknowledges the interdependence between the self and others, as well as a domain of situations in which self-interest and collective interest conflict (Kelley et al., 2003; Kelley \& Thibaut, 1978; Rusbult \& Van Lange, 2003). More generally, interdependence theory is a broad theory of the structure of interpersonal situations and how these situations afford the expression of certain motives among individuals. From this perspective, we propose two broad principles that are useful for understanding the effectiveness of incentives in social dilemmas.

A first principle focuses on the interpersonal motives of the self. People may be strongly focused on enhancing immediate outcomes for self (direct self-interest) but may also take into account broader considerations, such as the goal to minimize (absolute) differences in outcomes for self and other (egalitarianism or fairness), the goal to enhance outcomes for the collective, or the goal to enhance outcomes in the future. The idea that these broader considerations (referred to as transformations by Kelley et al., 2003) can shape behavior and interactions has received strong support in past research (for an overview, see Van Lange, De Cremer, van Dijk, \& Van Vugt, 2007). Specifically, this first principle, which we term the given matrix thesis, states that an individual's initial preferences are shaped by the direct concrete outcomes of an interaction for the self (e.g., the monetary payoffs for the self during social dilemmas). Although motives other than direct self-interest might come into play, interdependence theory suggests that self-interest represents a powerful motive in interdependence situations. This thesis is shared by other theories and frameworks and has the important implication that people should be responsive to structural changes in interdependence patterns underlying social dilemmas (Kelley et al., 2003; Olson, 1965; Yamagishi, 1986, 1988). ${ }^{2}$ Importantly, incentives can affect the structure of outcomes in the given matrix by enhancing the correspondence of outcomes and reducing the conflict of interests between participants in the dilemma. That is, when incentives are present, there is less discrepancy between self-interest and collective interests compared to

\footnotetext{
${ }^{2}$ The notion that self-interest represents a powerful motive is shared by classic theorizing in the social and behavioral sciences. Also, the assumption that other-regarding motives might also be relevant to explain behavior in interdependence situations is increasingly acknowledged in several contemporary models and theories of social preferences and human cooperation (e.g., Fehr \& Schmidt, 1999; Van Lange, 1999).
} 
when incentives are absent. Specifically, both rewards and punishments of equal magnitude will result in a similar effect, rendering cooperation in the self-interest of each individual in the dilemma (Rapoport \& Chammah, 1965; see also Komorita \& Parks, 1995). Indeed, prior research has suggested that reward of cooperation or punishment of noncooperation results in greater levels of cooperation in social dilemmas relative to when incentives are absent (Fehr \& Gächter, 2000; Mulder, 2008; Ostrom et al., 1992; Parks, 2000; Rand et al., 2009; Yamagishi, 1986). Thus, following the given matrix thesis of interdependence theory, incentives are predicted to increase cooperation (Hypothesis 1).

Interdependence theory extends many other theories by assuming that self-interest is not the only interpersonal motive that is relevant to interdependence situations, such as social dilemmas. It also assumes that people might adopt broader motives, including other-regarding motives, and that people are oriented toward understanding others' behavior in terms of such motives. Thus, a second principle focuses on the perceived interpersonal motives of interdependent others. This principle states not only that people might adopt broader motives, including other-regarding motives in situations of interdependence, but also that people are oriented toward understanding others' behavior in terms of such motives. For example, people are oriented toward understanding whether self-interest or other-regarding motives provide the most plausible account of behavior. To illustrate, strong deviations from selfinterested preferences by Mary that benefit John promote John's trust. This trust, in turn, may enhance John's pro-partner behavior, which may affect Mary's trust and pro-partner behavior as well. Such dynamics explain why trust and commitment in relationships can be maintained and grow over time (Wieselquist, Rusbult, Foster, \& Agnew, 1999; see also Murray \& Holmes, 2009). As we suggest below, this principle can help identify features of the situation that can make incentives more or less effective at enhancing cooperation.

\section{What Factors Promote the Effectiveness of Reward and Punishment?}

According to an interdependence-theoretical analysis, the perceived motives by others are likely to influence the effectiveness of incentives. Generally speaking, incentives should be more effective if the goals of those who administer incentives are perceived as aimed at enhancing collective interest, rather than their own interests. That is, incentives should be more effective to the degree that they are perceived as guided by cooperative motives (e.g., Kelley et al., 2003; Mulder \& Nelissen, 2011; Van Lange \& Rusbult, in press). This premise has implications for two variables and their impact on the incentives-cooperation relationship: (a) the cost of incentives and (b) the source of incentives.

\section{Cost of Incentives}

According to an interdependence perspective, incentives should be more effective to the extent that incentives are seen as genuine tools that others use to promote collective interests. When others are expending greater costs to provide reward (for cooperation) and deliver punishments (for noncooperation), people are likely to believe that these others seek to promote cooperation, rather than their self-interests. In fact, research suggests that people who are genuinely concerned about collective outcomes tend to sacrifice self-interest to provide punishment, with the punishment largely directed toward noncooperators (Egas \& Riedl, 2008; Fehr \& Gächter, 2000, 2002; Herrmann, Thöni, \& Gächter, 2008; Masclet, Noussair, Tucker, \& Villeval, 2003). That is, such punishment is costly to the punisher, who is not simply acting in self-interest. Thus, cooperation should increase as a function of group members' perceptions of a linkage between the costliness of incentives provided by others and those others' cooperative motives. We aim to provide more conclusive evidence relevant to the prediction that costly incentives will increase cooperation in social dilemmas, compared to when incentives are free to administer (Hypothesis 2).

\section{The Source of Incentives}

Past research has examined two distinct sources: decentralized incentives, which are provided by individuals who participate in the dilemma themselves and have equal roles, versus centralized incentives, which are provided by some kind of external force, often an authority figure (e.g., a government, manager, teacher, or the experimenter). Any difference in perceived cooperative motives between similar-status ingroup members and a central authority may influence the effectiveness of the incentive system.

In fact, power is often associated with self-interest motives. As power increases, people have been shown to be more concerned with their self-interest (Keltner, Langner, \& Allison, 2006), feel a sense of entitlement (De Cremer \& van Dijk, 2005, 2008), and tend to become less cooperative in social dilemmas (De Cremer \& van Dijk, 2005; Samuelson \& Allison, 1994; van Dijk \& De Cremer, 2006). Moreover, people in a position of (unilateral) power tend to be trusted less than those who hold equal power and have similar roles (Kramer, 1999; Muthusamy \& White, 2006; Van Vugt, Hogan, \& Kaiser, 2008). People are also more inclined to cooperate with ingroup members with similar status (e.g., Kalkhoff \& Barnum, 2000; Oldmeadow, Platow, Foddy, \& Anderson, 2003; Turner, 1991) and those who share similar characteristics (Parks, Sanna, \& Berel, 2001). Additionally, people tend to react more positively to power when this influence is mutual and not the result of a single power holder (Carpenter \& Matthews, 2009; Muthusamy \& White, 2006). Therefore, dispensers of incentives who are not part of the social dilemma may be seen as more strongly guided by self-interest motives (e.g., Komorita, Sheposh, \& Braver, 1968; Kramer, 1999; Mulder, Verboon, \& De Cremer, 2009), fueling the perception that they are administering an incentive for their own personal gains rather than concern for the collective outcome. Thus, we predict centralized incentive systems - provided by authority figures and not direct participantswill be less effective than decentralized incentive systems (Hypothesis 3 ).

\section{Overview of the Meta-Analysis}

We report a meta-analytic review of the effect of both rewards and punishment on cooperation in experimental social dilemmas. In so doing, we examine the above-noted predictions derived from interdependence theory. Specifically, we estimate the overall average effect of both rewards and punishments on cooperation and then test whether these relationships are moderated by the cost and source of incentives. Besides testing the interdependence perspec- 
tive, we also consider several other perspectives about the differences between reward and punishment. Specifically, we examine if punishments are more effective compared to rewards in the short term, while also considering the possibility that rewards are more effective than punishments in the long term. Last, we examine the moderating role of the type of dilemma, number of iterations, group size, cost-to-fine ratio, participant payment, partner matching, and country of participants.

\section{Method}

\section{Search for Studies}

We searched several databases for published studies, including PsycINFO, PsycARTICLES, Econlit, Google Scholar, Social Sciences Citation Index, Web of Science, Worldwide Political Science Abstracts, and Dissertations Online. We searched the entire text of English written journal articles by using the terms punishment, rewards, incentive, reinforcer, and sanction along with social dilemmas, public goods dilemmas, resource dilemmas, and voluntary contributions mechanism. We searched the references of all research and review articles for relevant studies. We also contacted over 150 researchers who attended the 2007 and 2009 International Conference for Social Dilemmas for published and unpublished data. We also posted a call for data to the Economic Science Association methods discussion group (http:// groups.google.com/group/esa-discuss). Finally, we contacted several authors who had published papers in the past 5 years with requests for additional data.

\section{Study Criteria}

There were several criteria for the selection of studies. First, studies had to be conducted on adult participants (ages 18 years and above). Second, all studies had to examine the effect of either a reward or punishment on cooperation in a social dilemma. We included only studies that compared a reward or punishment condition to a control condition and excluded conditions when both reward and punishments were used simultaneously. The incentives could have been delivered by a fine imposed by the experimenter, by providing participants an opportunity to sanction each other during the dilemma, or by a leader in the dilemma. The social dilemma could be either a public goods or give-some dilemma, prisoner's dilemma, resource or take-some dilemma, or other possible matrix games that included some degree of a conflict between individual and collective interests. Specifically, mutual cooperation had to yield higher outcomes than mutual defection, and according to the individual, defection always yielded a higher personal outcome than cooperation (Dawes, 1980). We excluded games that did not fit the strict criteria above, including ultimatum bargaining games, negotiations, trust games, and dictator games. ${ }^{3}$ We applied these criteria to evaluate potentially relevant studies, which uncovered a total of 103 papers. However, some papers were excluded because they either did not have a control condition or failed to report the statistics necessary to calculate the effect size. This resulted in a total of 76 papers that contained 187 effect sizes (150 published and 37 unpublished).

\section{Coding Procedure}

Cost of incentive: Costly versus free. Many experiments have participants make a payment to either punish or reward other group members. However, some experiments do not include a cost for providing incentives to fellow participants (e.g., voting if a player should be punished, experimenter provides a sanctioning system free of charge to the participants). Therefore, we coded whether the incentive was costly $(k=116)$, free $(k=70)$, or not determined $(k=1)$.

Source of incentives: Centralized versus decentralized. In social dilemma experiments with incentives, researchers often allow participants in the dilemma an option to either reward or punish their partners. During the first stage of these experiments, participants make their choices in the dilemma. In the second stage, participants are provided feedback about the choices of their partners and given the opportunity to pay or vote toward the end of rewarding or punishing other members. For example, a participant might pay $\$ 1$ to reduce the earnings of another member by $\$ 3$. In these experiments, punishment is confidential, and participants are not informed which of the other partners paid to reduce their own earnings. In other experiments, however, an incentive system is implemented by the experimenter that either rewards cooperators or punishes noncooperators (e.g., the earnings of the least cooperative member are reduced by a specific amount). This second scenario is much like a fine for noncooperation. These studies often vary in their probability of defection being detected. We coded if the incentive was decentralized, that is, delivered by the participants in the social dilemma $(k=102)$; centralized, meaning that an incentive mechanism (e.g., a fine) was imposed by the experimenter $(k=83)$; or not applicable $(k=2)$.

Cost-to-fine ratio in punishment studies. In many studies, punishment varied not only in cost to the punisher but also in the impact it had on the punished. In some studies, participants paid one monetary unit to decrease their partner's earnings by one monetary unit $(k=6)$. However, in other studies, one monetary unit reduced the partner's earnings by either two $(k=11)$, three $(k=59)$, or four $(k=5)$ monetary units. In several other studies, each monetary unit purchased a $10 \%$ reduction in a group member's earnings $(k=10)$.

Group size. We coded group size as a continuous variable. The mode of group size was a four-person group $(k=88)$. Excluding one outlier (a 1,000-person group), the mean of group

\footnotetext{
${ }^{3}$ We did not include these paradigms because they (a) do not share the same game theoretical or interdependence features (e.g., a dictator game represents unilateral dependence rather than interdependence), (b) add qualities that make the game different (e.g., most negotiation games include an element of coordination, represent incomplete information about their partner's preferences, may be time bound, and can be asymmetrical, in that the roles of the players differ), or (c) make salient psychological states or orientations that are somewhat different from social dilemmas (e.g., the trust game calls for trust, less so for cooperation; the ultimatum bargaining appears to challenge violations of fairness). Furthermore, they have been primarily used in a two-person format rather than an $n$-person format.
} 
size is a four-person group, and the range is between two- and 16-person groups.

Type of experimental protocol: Partner design versus stranger design. A common method of punishment protocol included in this meta-analysis is the paradigm developed by Fehr and Gächter (2000). Fehr and Gächter had participants play an iterated four-person public goods dilemma allowing participants an opportunity to punish their partners in the dilemma after each trial. Originally, Fehr and Gächter composed two conditions-the stranger design and the partner design. In the stranger design, participants play the same dilemma for several trials but are randomly assigned to a new group after each trial. In the partner design, however, participants remain anonymous but are not reassigned to a new group after each trial and remain in the same group for the entire experiment. Of the studies that employed the Fehr and Gächter protocol, we coded if the study used the partner design $(k=62)$ or the stranger design $(k=32)$.

One-shot versus iterated dilemma. In social dilemma studies, participants are allowed to interact only once, or the dilemma may occur repeatedly for several iterations. The sample of studies includes both one-shot $(k=54)$ and iterated $(k=133)$ dilemmas. We also coded the number of iterations as a continuous variable $(M=12, M d n=10$, mode $=10)$.

Type of dilemma. In this analysis, public goods dilemmas $(k=139)$ were the most common dilemma, followed by the prisoner's dilemma $(k=19)$ and the resource dilemma $(k=11)$. Also, a few effect sizes were from studies that used several of these dilemmas coded above $(k=6)$ or used a different type of social dilemma $(k=12)$. Additionally, the social dilemma paradigms in this sample of studies involved either decisions about real money $(k=168)$ or decisions in hypothetical scenarios $(k=19)$.

Country of participants. The studies included in our analysis were conducted in 27 different countries. Most studies were conducted in the United States $(k=63)$, the Netherlands $(k=48)$, Australia $(k=13)$, Switzerland $(k=13)$, England (9), Japan $(k=$ $7)$, and Russia $(k=6)$. Other countries represented include Austria, China, Greece, Israel, Saudi Arabia, Turkey, Ukraine, and several more. The country of each effect size is labeled in Table 1.

\section{Overview of Analysis}

We use the $d$ statistic as the measure of effect size. This value is the difference between the mean levels of cooperation in the treatment and control conditions divided by the pooled standard deviation. This is a common measure of effect size for examining the effect of a manipulated dichotomous variable (incentives vs. control) on a continuous dependent variable (degree of cooperation). A positive $d$ value indicates greater cooperation in the reward/punishment condition, relative to a control condition. When the means and standard deviations were not directly reported, the $d$ values were calculated using the sample size along with the $F$ score, $t$ value, or chi-square value (see Lipsey \& Wilson, 2001).

Several studies allowed us to code multiple effect sizes. For example, one study could include a control condition and then three separate punishment treatments, affording the calculation of three separate effect sizes. However, the effect sizes are nonindependent because they share several methodological features as well as the same control condition. Therefore, we applied Cooper's
(1998) shifting-units-of-analysis approach to handling nonindependent effect sizes when conducting analyses. Using this approach, we averaged over all the effects abstracted from a single study. This creates a single effect size for each study with multiple nonindependent effect sizes. These combined effect sizes are then used in each analysis.

To begin our analysis, we first estimate the average effect sizes for both punishment and reward with cooperation using a randomeffects model. A fixed-effects model is inappropriate because we assume that we do not have the entire population of studies and that there will be systematic between-study variation. Specifically, we are assuming that the effect of incentives on cooperation in social dilemmas will have systematic variation that will be explained, in part, by the study characteristics we mentioned above (see also Lipsey \& Wilson, 2001). We then assess the amount of estimated variation in the effect size distribution using several indicators of heterogeneity $\left(T, T^{2}\right.$, and $\left.I^{2}\right)$ and homogeneity $(Q)$ of variance. We then apply a mixed-effects model when examining moderators because a random-effects model assumes only random variation in the effect size distribution. However, one limitation of a mixed-effects model is that it may be too conservative and result in Type II errors, compared to a fixed-effects model (Lipsey \& Wilson, 2001). We report any discrepancies between fixed- and mixed-effects analyses. Finally, because several of the moderators are correlated, we conclude our analyses with a multiple regression model with several study characteristics predicting the effect size. Analyses were conducted using the Hedges and Olkin (1985) method with comprehensive meta-analysis.

\section{Results}

\section{The Effects of Punishment Versus Reward on Cooperation}

We begin our analysis by reporting the main effects of both punishment and rewards on cooperation in social dilemmas and then comparing the effectiveness between these incentives. Throughout the Results section, we report the effects of punishment first, and then rewards, because punishments have received the most attention in prior research and we have obtained a much larger sample of punishment effect sizes $(k=154)$ compared to reward effect sizes $(k=33)$.

Punishment and cooperation. Studies examining the punishment-cooperation relationship used in the meta-analysis, including their effect sizes and study characteristics, are listed in Table 1. Prior to analyses, we created a single averaged effect size for studies that reported multiple nonindependent effect sizes, which reduced the sample of effect sizes $(k=126)$. As expected, punishment had a medium-sized, positive effect on cooperation in social dilemmas $(d=0.70,95 \%$ confidence interval $[\mathrm{CI}]$ lower limit $[L L]=0.60$, upper limit $[U L]=0.80$, 90\% prediction interval $L L=0.18, U L=1.58$ ). The indicators of heterogeneity of the effect size distribution suggest that there is variation in the true effect size distribution $\left(T^{2}=.28, T=.53\right)$ and that a substantial amount of this variation can be explained by between-study differences $\left(I^{2}=90.55 \%\right)$. Moreover, the overall punishmentcooperation effect size distribution contained more variation than would be expected by chance, $Q(125)=1,322, p<.001$. 
Table 1

Studies on the Punishment-Cooperation Relationship

\begin{tabular}{|c|c|c|c|c|c|c|c|c|c|c|c|}
\hline Study & $N$ & $\mathrm{CO}$ & $d$ & $L L / U L$ & DV & GS & $\mathrm{IT}(\#)$ & $\mathrm{SD} / \mathrm{PM}$ & $\mathrm{DC} / \mathrm{C}$ & $\$ / \mathrm{Fr}$ & $\mathrm{P} / \mathrm{NP}$ \\
\hline Bell, Petersen, \& Hautaluoma (1989) & 60 & US & 0.22 & $-0.30 / 0.74$ & RD & 3 & $\mathrm{IT}(7)$ & - & $\mathrm{C}$ & $\mathrm{Fr}$ & $\mathrm{P}$ \\
\hline Bochet, Page, \& Putterman (2006) & 116 & US & 0.96 & $0.57 / 1.70$ & PGD & 4 & $\mathrm{IT}(10)$ & PM & DC & $\$$ & $\mathrm{P}$ \\
\hline Bornstein \& Weisel (2010a) & 72 & IS & 1.42 & $0.76 / 2.07$ & PGD & 4 & IT(18) & PM & DC & $\$$ & $\mathrm{P}$ \\
\hline Sample b & 72 & IS & 0.52 & $0.03 / 1.01$ & PGD & 4 & IT(18) & PM & DC & $\$$ & $\mathrm{P}$ \\
\hline Bornstein \& Weisel (2010b) & 72 & IS & 1.65 & $0.94 / 2.36$ & PGD & 4 & IT(18) & PM & DC & $\$$ & $\mathrm{P}$ \\
\hline Sample b & 72 & IS & 0.71 & $0.20 / 1.23$ & PGD & 4 & IT(18) & PM & DC & $\$$ & $\mathrm{P}$ \\
\hline Caldwell (1976) & 130 & US & 0.48 & $0.13 / 0.83$ & PD & 5 & IT(40) & - & DC & $\mathrm{Fr}$ & NP \\
\hline Camera \& Casari (2009) & 80 & US & 0.62 & $-0.24 / 1.07$ & PD & 2 & $\operatorname{IT}(100)$ & - & DC & $\$$ & $\mathrm{P}$ \\
\hline Carpenter (2007b) & 46 & US & 0.55 & $0.14 / 0.97$ & PGD & 7 & $\operatorname{IT}(10)$ & SD & $\mathrm{C}$ & $\$$ & $\mathrm{P}$ \\
\hline Sample b & 46 & US & 0.44 & $0.02 / 0.85$ & PGD & 7 & $\mathrm{IT}(10)$ & SD & $\mathrm{C}$ & $\$$ & $\mathrm{P}$ \\
\hline Sample c & 46 & US & 0.18 & $-0.23 / 0.59$ & PGD & 7 & $\mathrm{IT}(10)$ & SD & $\mathrm{C}$ & $\$$ & $\mathrm{P}$ \\
\hline Carpenter \& Matthews (2009) & 100 & US & 0.59 & $0.19 / 0.99$ & PGD & 4 & IT(10) & PM & DC & $\$$ & $\mathrm{P}$ \\
\hline \multicolumn{12}{|l|}{ Carpenter, Matthews, \& Ong'ong'a } \\
\hline$(2004)$ & 72 & US & 0.94 & $0.45 / 1.44$ & PGD & 4 & $\mathrm{IT}(10)$ & $\mathrm{PM}$ & DC & $\$$ & $\mathrm{P}$ \\
\hline Casari \& Luini (2009) & 60 & IT & 1.60 & $0.30 / 1.27$ & PGD & 5 & IT(10) & PM & DC & $\$$ & $\mathrm{P}$ \\
\hline Chen, Pillutla, \& Yao (2009) Study 2 & 50 & US & 0.52 & $-0.05 / 1.08$ & PGD & 4 & OS & - & $\mathrm{C}$ & $\mathrm{Fr}$ & NP \\
\hline Cinyabuguma, Page, \& Putterman & & & & & & & & & & & \\
\hline (2005) & 128 & US & 0.96 & $0.12 / 1.80$ & PGD & 16 & IT(15) & - & DC & $\$$ & $\mathrm{P}$ \\
\hline Dickinson (2001) & 80 & US & 1.26 & $0.78 / 1.74$ & PGD & 4 & $\mathrm{IT}(8)$ & - & $\mathrm{C}$ & Fr & $\mathrm{P}$ \\
\hline Sample b & 80 & US & 1.29 & $0.81 / 1.77$ & PGD & 4 & IT(8) & - & $\mathrm{C}$ & $\mathrm{Fr}$ & $\mathrm{P}$ \\
\hline \multicolumn{12}{|l|}{ Dreber, Rand, Fudenberg, \& Nowak } \\
\hline$(2008)$ & 52 & US & 0.78 & $0.71 / 1.45$ & PD & 2 & IT(24) & - & DC & $\$$ & $\mathrm{P}$ \\
\hline Sample b & 52 & US & 0.37 & $-0.78 / 0.98$ & PD & 2 & $\mathrm{IT}(24)$ & - & DC & $\$$ & $\mathrm{P}$ \\
\hline \multicolumn{12}{|l|}{ Eek, Loukopoulos, Satoshi, \& Gärling } \\
\hline$(2002)$ & 208 & $S E$ & 2.16 & $1.56 / 2.75$ & PD & 1,000 & OS & - & $\mathrm{C}$ & $\mathrm{Fr}$ & NP \\
\hline Egas \& Riedl (2008) & 324 & NL & 0.08 & $-0.14 / 0.31$ & PGD & 3 & IT(6) & SD & DC & $\$$ & $\mathrm{P}$ \\
\hline Sample b & 324 & NL & 0.50 & $0.27 / 0.72$ & PGD & 3 & IT(6) & SD & DC & $\$$ & $\mathrm{P}$ \\
\hline Sample c & 306 & NL & 0.21 & $-0.01 / 0.44$ & PGD & 3 & $\operatorname{IT}(6)$ & SD & DC & $\$$ & $\mathrm{P}$ \\
\hline Sample d & 324 & NL & -0.09 & $-0.31 / 0.13$ & PGD & 3 & $\mathrm{IT}(6)$ & SD & DC & $\$$ & $\mathrm{P}$ \\
\hline Etran, Page, \& Putterman (2009) & 80 & US & 0.37 & $0.14 / 0.59$ & PGD & 4 & IT(3) & PM & DC & $\$$ & $\mathrm{P}$ \\
\hline Fehr \& Gächter (2000) & 24 & $\mathrm{CH}$ & 1.35 & $0.46 / 2.23$ & PGD & 4 & $\mathrm{IT}(10)$ & SD & DC & $\$$ & $\mathrm{P}$ \\
\hline Sample b & 80 & $\mathrm{CH}$ & 1.65 & $1.14 / 2.15$ & PGD & 4 & $\mathrm{IT}(10)$ & $\mathrm{PM}$ & DC & $\$$ & $\mathrm{P}$ \\
\hline Fehr \& Gächter (2002) & 236 & $\mathrm{CH}$ & 1.35 & $1.17 / 1.53$ & PGD & 4 & IT(6) & SD & DC & $\$$ & $\mathrm{P}$ \\
\hline Fuster \& Meier (2010) & 15 & US & 1.10 & $0.50 / 1.74$ & PGD & 4 & $\mathrm{IT}(6)$ & PM & DC & $\$$ & $\mathrm{P}$ \\
\hline Sample b & 19 & US & 0.94 & $0.40 / 1.48$ & PGD & 4 & IT(6) & PM & DC & $\$$ & $\mathrm{P}$ \\
\hline Gächter \& Herrmann (2009) & 141 & $\mathrm{CH}$ & 0.31 & $0.14 / 0.48$ & PGD & 3 & OS & SD & DC & $\$$ & $\mathrm{P}$ \\
\hline Sample b & 102 & $\mathrm{CH}$ & 0.30 & $0.11 / 0.50$ & PGD & 3 & OS & SD & DC & $\$$ & $\mathrm{P}$ \\
\hline Sample c & 180 & RU & -0.05 & $-0.20 / 0.09$ & PGD & 3 & OS & SD & DC & $\$$ & $\mathrm{P}$ \\
\hline Sample d & 180 & RU & -0.08 & $-0.23 / 0.06$ & PGD & 3 & OS & SD & DC & $\$$ & $\mathrm{P}$ \\
\hline Gächter \& Herrmann (2011) & 205 & RU & -0.07 & $-0.21 / 0.07$ & PGD & 3 & OS & SD & DC & $\$$ & $\mathrm{P}$ \\
\hline Sample b & 105 & RU & 0.07 & $-0.13 / 0.26$ & PGD & 3 & OS & SD & DC & $\$$ & $\mathrm{P}$ \\
\hline Sample c & 143 & RU & 0.18 & $0.01 / 0.36$ & PGD & 3 & OS & SD & DC & $\$$ & $\mathrm{P}$ \\
\hline Sample d & 153 & RU & -0.17 & $-0.33 /-0.01$ & PGD & 3 & OS & SD & DC & $\$$ & $\mathrm{P}$ \\
\hline Gächter, Renner, \& Sefton (2008) & 105 & $\mathrm{CH}$ & 0.71 & $0.31 / 1.11$ & PGD & 3 & $\mathrm{IT}(10)$ & PM & DC & $\$$ & $\mathrm{P}$ \\
\hline Sample b & 102 & $\mathrm{CH}$ & 1.95 & $1.48 / 2.42$ & PGD & 3 & $\mathrm{IT}(50)$ & PM & DC & $\$$ & $\mathrm{P}$ \\
\hline Gächter \& Thöni (2005) & 51 & $\mathrm{CH}$ & 1.85 & $1.19 / 2.50$ & PGD & 3 & $\mathrm{IT}(10)$ & SD & DC & $\$$ & $\mathrm{P}$ \\
\hline Sample a & 126 & $\mathrm{CH}$ & 0.20 & $-0.15 / 0.56$ & PGD & 3 & $\mathrm{IT}(10)$ & SD & DC & $\$$ & $\mathrm{P}$ \\
\hline Sample b & 54 & $\mathrm{CH}$ & 0.62 & $0.07 / 1.16$ & PGD & 3 & $\mathrm{IT}(10)$ & SD & DC & $\$$ & $\mathrm{P}$ \\
\hline Herrmann, Thöni, \& Gächter (2008) & 56 & US & 1.36 & $1.00 / 1.72$ & PGD & 4 & $\mathrm{IT}(10)$ & PM & DC & $\$$ & $\mathrm{P}$ \\
\hline Study 2 & 56 & UK & 1.38 & $1.02 / 1.75$ & PGD & 4 & $\mathrm{IT}(10)$ & PM & DC & $\$$ & $\mathrm{P}$ \\
\hline Study 3 & 68 & DK & 1.12 & $0.81 / 1.42$ & PGD & 4 & $\mathrm{IT}(10)$ & PM & DC & $\$$ & $\mathrm{P}$ \\
\hline Study 4 & 60 & DE & 1.42 & $0.82 / 1.47$ & PGD & 4 & $\mathrm{IT}(10)$ & PM & DC & $\$$ & $\mathrm{P}$ \\
\hline Study 5 & 48 & $\mathrm{CH}$ & 1.87 & $1.40 / 2.34$ & PGD & 4 & $\mathrm{IT}(10)$ & PM & DC & $\$$ & $\mathrm{P}$ \\
\hline Study 6 & 96 & $\mathrm{CH}$ & 1.21 & $0.95 / 1.48$ & PGD & 4 & $\mathrm{IT}(10)$ & PM & DC & $\$$ & $\mathrm{P}$ \\
\hline Study 7 & 68 & BY & 0.55 & $0.29 / 0.80$ & PGD & 4 & IT(10) & PM & DC & $\$$ & $\mathrm{P}$ \\
\hline Study 8 & 44 & UA & 0.08 & $-0.22 / 0.38$ & PGD & 4 & $\mathrm{IT}(10)$ & PM & DC & $\$$ & $\mathrm{P}$ \\
\hline Study 9 & 152 & RU & 0.39 & $0.23 / 0.56$ & PGD & 4 & IT(10) & PM & DC & $\$$ & $\mathrm{P}$ \\
\hline Study 10 & 44 & GR & -0.16 & $-0.46 / 0.14$ & PGD & 4 & IT(10) & PM & DC & $\$$ & $\mathrm{P}$ \\
\hline Study 11 & 64 & TR & 0.33 & $0.08 / 0.58$ & PGD & 4 & $\mathrm{IT}(10)$ & PM & DC & $\$$ & $\mathrm{P}$ \\
\hline Study 12 & 48 & SA & -0.15 & $-0.43 / 0.14$ & PGD & 4 & IT(10) & PM & DC & $\$$ & $\mathrm{P}$ \\
\hline Study 13 & 52 & OM & -0.01 & $-0.28 / 0.27$ & PGD & 4 & IT(10) & PM & DC & $\$$ & $\mathrm{P}$ \\
\hline Study 14 & 84 & $\mathrm{KR}$ & 1.64 & $1.31 / 1.97$ & PGD & 4 & $\mathrm{IT}(10)$ & PM & DC & $\$$ & $\mathrm{P}$ \\
\hline Study 15 & 96 & $\mathrm{CN}$ & 1.23 & $0.96 / 1.49$ & PGD & 4 & IT(10) & PM & DC & $\$$ & $\mathrm{P}$ \\
\hline Study 16 & 40 & $\mathrm{AU}$ & 2.14 & $1.58 / 2.71$ & PGD & 4 & IT(10) & PM & DC & $\$$ & $\mathrm{P}$ \\
\hline
\end{tabular}


Table 1 (continued)

\begin{tabular}{|c|c|c|c|c|c|c|c|c|c|c|c|}
\hline Study & $N$ & $\mathrm{CO}$ & $d$ & $L L / U L$ & DV & GS & $\mathrm{IT}(\#)$ & $\mathrm{SD} / \mathrm{PM}$ & $\mathrm{DC} / \mathrm{C}$ & $\$ / F r$ & $\mathrm{P} / \mathrm{NP}$ \\
\hline Hopfensitz \& Rueben (2009) & 81 & NL & 1.08 & $0.47 / 1.70$ & PD & 2 & OS & - & DC & $\$$ & $\mathrm{P}$ \\
\hline Kieruj, Mulder, \& Nelissen (2008) & 56 & NL & 2.80 & $2.07 / 3.54$ & PGD & 4 & $\mathrm{IT}(6)$ & - & $\mathrm{C}$ & $\mathrm{Fr}$ & $\mathrm{P}$ \\
\hline Kocher, Martinsoon, \& Visser (2008) & 120 & ZA & 0.22 & $0.03 / 0.40$ & PGD & 3 & OS & SD & DC & $\$$ & $\mathrm{P}$ \\
\hline Komorita \& Barth (1985) & 60 & US & -0.21 & $-0.78 / 0.38$ & PD & 3 & $\mathrm{IT}(24)$ & - & $\mathrm{C}$ & Fr & $\mathrm{P}$ \\
\hline Kroll, Cherry, \& Shogren (2007) & 35 & US & 0.88 & $0.49 / 1.27$ & PGD & 5 & $\mathrm{IT}(10)$ & PM & $\mathrm{DC}$ & $\$$ & $\mathrm{P}$ \\
\hline Martichuski \& Bell (1991) & 48 & US & 0.66 & $0.08 / 1.24$ & RD & 3 & $\mathrm{IT}(15)$ & - & $\mathrm{C}$ & $\mathrm{Fr}$ & $\mathrm{P}$ \\
\hline McCusker \& Carnevale (1995) & 124 & US & 0.82 & $0.43 / 1.20$ & $\mathrm{RD}$ & 8 & IT(12) & - & $\mathrm{C}$ & $\$$ & $\mathrm{P}$ \\
\hline Study 2 & 48 & US & 0.66 & $0.04 / 1.27$ & RD & 8 & $\mathrm{IT}(12)$ & - & $\mathrm{C}$ & $\$$ & $\mathrm{P}$ \\
\hline Mulder (2005) & 154 & NL & 0.58 & $0.26 / 0.90$ & PGD & 4 & os & - & $\mathrm{C}$ & $\mathrm{Fr}$ & $\mathrm{P}$ \\
\hline Mulder (2008) & 76 & NL & 0.47 & $0.02 / 0.93$ & PGD & 4 & OS & - & $\mathrm{C}$ & $\mathrm{Fr}$ & $\mathrm{P}$ \\
\hline $\begin{array}{l}\text { Mulder, van Dijk, De Cremer, \& Wilke } \\
\text { (2001) }\end{array}$ & 100 & NL & 0.45 & $0.05 / 0.84$ & PGD & 4 & OS & - & $\mathrm{C}$ & $\mathrm{Fr}$ & $\mathrm{P}$ \\
\hline Sample b & 38 & NL & 0.73 & $0.07 / 1.38$ & PGD & 4 & OS & - & $\mathrm{C}$ & Fr & $\mathrm{P}$ \\
\hline $\begin{array}{l}\text { Mulder, van Dijk, De Cremer, \& Wilke } \\
\text { (2002) }\end{array}$ & 106 & NL & 0.53 & $0.15 / 0.92$ & PGD & 4 & OS & - & $\mathrm{C}$ & $\mathrm{Fr}$ & $\mathrm{P}$ \\
\hline $\begin{array}{l}\text { Mulder, van Dijk, De Cremer, \& Wilke } \\
\text { (2003) }\end{array}$ & 126 & NL & 0.35 & $-0.01 / 0.71$ & PGD & 4 & OS & - & $\mathrm{C}$ & $\mathrm{Fr}$ & $\mathrm{P}$ \\
\hline $\begin{array}{l}\text { Mulder, van Dijk, De Cremer, \& Wilke } \\
\quad \text { (2006a) } \\
\text { Study } 2\end{array}$ & $\begin{array}{l}113 \\
159\end{array}$ & $\begin{array}{l}\text { NL } \\
\text { NL }\end{array}$ & $\begin{array}{l}0.90 \\
0.59\end{array}$ & $\begin{array}{l}0.51 / 1.28 \\
0.27 / 0.91\end{array}$ & $\begin{array}{l}\text { PGD } \\
\text { PGD }\end{array}$ & $\begin{array}{l}4 \\
4\end{array}$ & $\begin{array}{l}\text { OS } \\
\text { OS }\end{array}$ & - & $\begin{array}{l}\mathrm{C} \\
\mathrm{C}\end{array}$ & $\begin{array}{l}\mathrm{Fr} \\
\mathrm{Fr}\end{array}$ & $\begin{array}{l}\mathrm{P} \\
\mathrm{P}\end{array}$ \\
\hline Mulder, van Dijk, De Cremer, \& Wilke & & & & & & & & & & & \\
\hline$(2006 b)$ & 50 & NL & 0.62 & $0.05 / 1.18$ & PGD & 4 & OS & - & $\mathrm{C}$ & $\mathrm{Fr}$ & $\mathrm{P}$ \\
\hline Study 2 & 123 & NL & 0.69 & $0.33 / 1.06$ & PGD & 4 & OS & - & $\mathrm{C}$ & $\mathrm{Fr}$ & $\mathrm{P}$ \\
\hline Sample b & 80 & NL & 0.39 & $-0.05 / 0.83$ & PGD & 4 & OS & - & $\mathrm{C}$ & $\mathrm{Fr}$ & $\mathrm{P}$ \\
\hline Study 3 & 100 & NL & 0.53 & $0.13 / 0.93$ & PGD & 4 & OS & - & $\mathrm{C}$ & $\mathrm{Fr}$ & $\mathrm{P}$ \\
\hline $\begin{array}{l}\text { Mulder, van Dijk, Wilke, \& De Cremer } \\
\text { (2005) }\end{array}$ & 124 & NL & 0.05 & $-0.30 / 0.41$ & PGD & 4 & OS & - & $\mathrm{C}$ & $\mathrm{Fr}$ & $\mathrm{P}$ \\
\hline Mulder \& van Prooijen (2006) & 154 & NL & 0.48 & $0.16 / 0.80$ & PGD & 4 & OS & - & $\mathrm{C}$ & Fr & $\mathrm{P}$ \\
\hline Myers (2009) & 72 & US & 1.12 & $0.63 / 1.62$ & PGD & 4 & $\mathrm{IT}(20)$ & $\mathrm{SD}$ & DC & $\$$ & $\mathrm{P}$ \\
\hline Nelissen \& Mulder (2010) & 56 & NL & 2.80 & $2.07 / 3.54$ & PGD & 4 & $\mathrm{IT}(6)$ & PM & DC & $\mathrm{Fr}$ & $\mathrm{P}$ \\
\hline Nikiforakis (2008) & 144 & $\mathrm{AU}$ & 1.03 & $0.67 / 1.40$ & PGD & 4 & $\mathrm{IT}(10)$ & SD & DC & $\$$ & $\mathrm{P}$ \\
\hline Sample b & 144 & $\mathrm{AU}$ & 1.63 & $1.23 / 2.04$ & PGD & 4 & $\mathrm{IT}(10)$ & PM & DC & $\$$ & $\mathrm{P}$ \\
\hline Sample c & 144 & $\mathrm{AU}$ & 0.21 & $-0.14 / 0.56$ & PGD & 4 & $\mathrm{IT}(10)$ & SD & DC & $\$$ & $\mathrm{P}$ \\
\hline Sample d & 144 & $\mathrm{AU}$ & 0.51 & $0.16 / 0.86$ & PGD & 4 & $\mathrm{IT}(10)$ & PM & DC & $\$$ & $\mathrm{P}$ \\
\hline Nikiforakis \& Normann (2008) & 48 & $\mathrm{AU}$ & 0.50 & $-0.07 / 1.08$ & PGD & 4 & $\mathrm{IT}(10)$ & $\mathrm{PM}$ & DC & $\$$ & $\mathrm{P}$ \\
\hline Sample b & 48 & $\mathrm{AU}$ & 1.09 & $0.49 / 1.70$ & PGD & 4 & IT(10) & PM & DC & $\$$ & $\mathrm{P}$ \\
\hline Sample c & 48 & $\mathrm{AU}$ & 2.07 & $1.37 / 2.77$ & PGD & 4 & $\mathrm{IT}(10)$ & PM & DC & $\$$ & $\mathrm{P}$ \\
\hline Sample d & 48 & $\mathrm{AU}$ & 2.24 & $1.52 / 2.97$ & PGD & 4 & $\mathrm{IT}(10)$ & $\mathrm{PM}$ & DC & $\$$ & $\mathrm{P}$ \\
\hline Nikiforakis, Normann, \& Wallace & & & & & & & & & & & \\
\hline (2010) & 44 & AU & 1.09 & $0.45 / 1.72$ & PGD & 4 & IT(10) & PM & DC & $\$$ & $\mathrm{P}$ \\
\hline Sample b & 48 & $\mathrm{AU}$ & 1.20 & $0.58 / 1.81$ & PGD & 4 & $\operatorname{IT}(10)$ & $\mathrm{PM}$ & DC & $\$$ & $\mathrm{P}$ \\
\hline Sample c & 44 & AU & 1.84 & $1.14 / 2.55$ & PGD & 4 & IT(10) & PM & DC & $\$$ & $\mathrm{P}$ \\
\hline Sample d & 48 & AU & 1.85 & $1.18 / 2.53$ & PGD & 4 & $\mathrm{IT}(10)$ & PM & DC & $\$$ & $\mathrm{P}$ \\
\hline O'Gorman, Henrich, \& Van Vugt & & & & & & & & & & & \\
\hline$(2008)$ & 44 & UK & 0.35 & $0.05 / 0.66$ & PGD & 4 & $\mathrm{IT}(6)$ & SD & DC & $\$$ & $\mathrm{P}$ \\
\hline Sample b & 44 & UK & -0.06 & $-0.36 / 0.24$ & PGD & 4 & IT(6) & SD & $\mathrm{C}$ & $\$$ & $\mathrm{P}$ \\
\hline O’Gorman \& Van Vugt (2010) & 48 & UK & 0.80 & $0.45 / 1.13$ & PGD & 4 & IT(6) & SD & $\mathrm{DC}$ & $\$$ & $\mathrm{P}$ \\
\hline Sample b & 44 & UK & 0.38 & $0.08 / 0.69$ & PGD & 4 & $\mathrm{IT}(6)$ & SD & $\mathrm{C}$ & $\$$ & $\mathrm{P}$ \\
\hline Page, Putterman, \& Unel (2005) & 128 & US & 1.29 & $0.91 / 1.67$ & PGD & 4 & $\operatorname{IT}(20)$ & PM & DC & $\$$ & $\mathrm{P}$ \\
\hline Patel, Cartwright, \& Van Vugt (2010) & 66 & UK & 0.20 & $-0.39 / 0.80$ & PGD & 4 & $\mathrm{IT}(10)$ & $\mathrm{PM}$ & DC & $\$$ & $\mathrm{P}$ \\
\hline Sample b & 36 & UK & 0.38 & $-0.45 / 1.21$ & PGD & 4 & IT(10) & PM & DC & $\$$ & $\mathrm{P}$ \\
\hline Sample c & 72 & UK & 0.21 & $-0.36 / 0.78$ & PGD & 4 & $\mathrm{IT}(10)$ & PM & DC & $\$$ & $\mathrm{P}$ \\
\hline Sample d & 36 & UK & -0.79 & $-1.71 / 0.13$ & PGD & 4 & $\operatorname{IT}(10)$ & PM & DC & $\$$ & $\mathrm{P}$ \\
\hline Rand, Dreber, Ellingsen, Fudenberg, \& & & & & & & & & & & & \\
\hline Nowak (2009) & 26 & US & 1.61 & $0.71 / 2.52$ & PGD & 4 & $\mathrm{IT}(50)$ & $\mathrm{PM}$ & DC & $\$$ & $\mathrm{P}$ \\
\hline Rapoport \& Au (2001) & 30 & HK & 0.58 & $-0.20 / 1.37$ & RD & 5 & $\mathrm{IT}(90)$ & - & $\mathrm{C}$ & $\mathrm{Fr}$ & $\mathrm{P}$ \\
\hline Reuben \& Riedl (2009) & 39 & NL & 4.51 & $3.33 / 5.70$ & PGD & 3 & $\operatorname{IT}(10)$ & PM & DC & $\$$ & $\mathrm{P}$ \\
\hline Sample b & 39 & NL & 0.90 & $0.24 / 1.56$ & PGD & 3 & IT(10) & PM & DC & $\$$ & $\mathrm{P}$ \\
\hline Sample c & 42 & NL & 2.38 & $1.59 / 3.17$ & PGD & 3 & IT(10) & PM & DC & $\$$ & $\mathrm{P}$ \\
\hline Sample d & 39 & NL & 1.77 & $1.00 / 2.54$ & PGD & 3 & IT(10) & PM & DC & $\$$ & $\mathrm{P}$ \\
\hline Riedl \& Ule (2009) & 12 & NL & 0.88 & $-0.49 / 2.24$ & PD & 6 & $\mathrm{IT}(60)$ & - & DC & $\$$ & $\mathrm{P}$ \\
\hline Sample b & 12 & NL & 0.81 & $-0.54 / 2.15$ & PD & 2 & $\mathrm{IT}(60)$ & - & DC & $\$$ & $\mathrm{P}$ \\
\hline Sample c & 13 & NL & 1.87 & $-0.17 / 3.90$ & PD & 2 & $\mathrm{IT}(60)$ & - & DC & $\$$ & $\mathrm{P}$ \\
\hline Sample d & 11 & NL & 0.68 & $-0.70 / 2.05$ & PD & 2 & $\mathrm{IT}(60)$ & - & DC & $\$$ & $\mathrm{P}$ \\
\hline Sato $(1987)$ & 36 & JP & 1.97 & $1.18 / 2.77$ & $\mathrm{RD}$ & 4 & $\mathrm{IT}(12)$ & - & $\mathrm{C}$ & $\mathrm{Fr}$ & $\mathrm{P}$ \\
\hline Sefton, Shupp, \& Walker (2007) & 18 & US & 0.32 & $-0.15 / 0.79$ & PGD & 4 & $\mathrm{IT}(10)$ & $\mathrm{PM}$ & $\mathrm{C}$ & $\mathrm{Fr}$ & $\mathrm{P}$ \\
\hline
\end{tabular}


Table 1 (continued)

\begin{tabular}{|c|c|c|c|c|c|c|c|c|c|c|c|}
\hline Study & $N$ & $\mathrm{CO}$ & $d$ & $L L / U L$ & DV & GS & $\mathrm{IT}(\#)$ & $\mathrm{SD} / \mathrm{PM}$ & $\mathrm{DC} / \mathrm{C}$ & $\$ / F r$ & $\mathrm{P} / \mathrm{NP}$ \\
\hline Sell \& Wilson (1999) & 64 & US & 0.70 & $0.36 / 1.02$ & PGD & 4 & $\operatorname{IT}(22)$ & - & NA & NA & $\mathrm{P}$ \\
\hline Shaw (1976) & 160 & US & 0.42 & $0.11 / 0.73$ & PD & 2 & $\mathrm{IT}(20)$ & - & NA & $\mathrm{Fr}$ & $\mathrm{P}$ \\
\hline Shinada \& Yamagishi (2007) & 106 & JP & 0.91 & $0.51 / 1.31$ & PD & 3 & OS & - & $\mathrm{C}$ & $\mathrm{Fr}$ & $\mathrm{P}$ \\
\hline Sample b & 103 & JP & 0.50 & $0.11 / 0.89$ & PD & 3 & OS & - & DC & $\$$ & $\mathrm{P}$ \\
\hline Study 2 & 96 & JP & 0.68 & $0.27 / 1.09$ & PD & 3 & OS & - & DC & $\$$ & $\mathrm{P}$ \\
\hline Sample b & 96 & JP & 0.34 & $-0.06 / 0.74$ & PD & 3 & OS & - & DC & $\$$ & $\mathrm{P}$ \\
\hline Sutter, Haigner, \& Kocher (2010) & 160 & AT & 1.08 & $0.74 / 1.43$ & PGD & 4 & $\mathrm{IT}(10)$ & - & DC & $\$$ & $\mathrm{P}$ \\
\hline Sample b & 165 & AT & 0.75 & $0.43 / 1.43$ & PGD & 4 & $\mathrm{IT}(10)$ & - & DC & $\$$ & $\mathrm{P}$ \\
\hline Sutter, Linder, \& Platsch (2009) & 120 & AT & 0.43 & $0.04 / 0.81$ & PGD & 4 & IT(10) & - & DC & $\$$ & $\mathrm{P}$ \\
\hline Tan (2008) & 48 & NL & 1.33 & $0.71 / 1.96$ & PGD & 4 & $\mathrm{IT}(15)$ & PM & DC & $\$$ & $\mathrm{P}$ \\
\hline Tenbrunsel \& Messick (1999) & 84 & US & -0.43 & $-0.87 / 0.00$ & PD & 2 & OS & - & $\mathrm{C}$ & Fr & NP \\
\hline Study 2 & 56 & US & -0.67 & $-1.21 /-0.13$ & PGD & NA & OS & - & $\mathrm{C}$ & $\mathrm{Fr}$ & NP \\
\hline Study 3 & 90 & US & 0.42 & $-0.10 / 0.94$ & PGD & NA & OS & - & $\mathrm{C}$ & $\mathrm{Fr}$ & NP \\
\hline Sample b & 90 & US & -0.23 & $-0.69 / 0.24$ & PGD & NA & OS & - & $\mathrm{C}$ & $\mathrm{Fr}$ & NP \\
\hline Tyran \& Feld (2004) & 84 & $\mathrm{CH}$ & 0.00 & $-0.43 / 0.43$ & $\mathrm{O}$ & 3 & OS & - & DC & Fr & $\mathrm{P}$ \\
\hline $\begin{array}{l}\text { van Prooíjen, Gallucci, \& Toeset } \\
\text { (2008) }\end{array}$ & 80 & NL & -0.20 & $-0.64 / 0.24$ & $\mathrm{O}$ & 4 & IT(5) & - & $\mathrm{C}$ & Fr & NP \\
\hline Sample b & 80 & NL & -0.91 & $-1.37 /-0.44$ & $\mathrm{O}$ & 4 & $\operatorname{IT}(5)$ & - & $\mathrm{C}$ & $\mathrm{Fr}$ & NP \\
\hline Study 2 & 36 & NL & 0.20 & $-0.45 / 0.86$ & $\mathrm{O}$ & 4 & IT(5) & - & $\mathrm{C}$ & $\mathrm{Fr}$ & $\mathrm{P}$ \\
\hline Sample b & 36 & NL & -0.78 & $-1.46 /-0.10$ & $\mathrm{O}$ & 4 & IT(5) & - & $\mathrm{C}$ & $\mathrm{Fr}$ & NP \\
\hline Van Vugt \& De Cremer (1999) & 90 & UK & 0.76 & $0.33 / 1.19$ & PGD & 6 & IT(4) & - & $\mathrm{C}$ & $\mathrm{Fr}$ & $\mathrm{P}$ \\
\hline Walker \& Halloran (2004) & 48 & US & -0.04 & $-0.32 / 0.25$ & PGD & 4 & OS & SD & DC & Fr & $\mathrm{P}$ \\
\hline Sample b & 48 & US & 0.11 & $-0.18 / 0.39$ & PGD & 4 & OS & SD & DC & $\mathrm{Fr}$ & $\mathrm{P}$ \\
\hline Wit \& Wilke (1990) & 61 & NL & 0.52 & $-0.01 / 1.06$ & PGD & 10 & OS & - & $\mathrm{C}$ & $\mathrm{Fr}$ & NP \\
\hline Study 2 & 120 & NL & 0.08 & $-0.34 / 0.51$ & PGD & 10 & OS & - & $\mathrm{C}$ & $\mathrm{Fr}$ & NP \\
\hline Study 3 & 120 & NL & 0.00 & $-0.36 / 0.36$ & PGD & 10 & OS & - & $\mathrm{C}$ & $\mathrm{Fr}$ & NP \\
\hline Study 4 & 63 & NL & 0.61 & $0.08 / 1.14$ & PGD & 10 & OS & - & $\mathrm{C}$ & $\mathrm{Fr}$ & NP \\
\hline Xiao \& Kunreuther (2010) & 63 & US & 0.31 & $-0.19 / 0.81$ & $\mathrm{O}$ & 2 & $\mathrm{IT}(10)$ & PM & $\mathrm{C}$ & $\$$ & $\mathrm{P}$ \\
\hline Sample b & 55 & US & 0.86 & $0.30 / 1.42$ & $\mathrm{O}$ & 2 & $\mathrm{IT}(10)$ & PM & $\mathrm{C}$ & $\$$ & $\mathrm{P}$ \\
\hline Sample c & 47 & US & 0.47 & $-0.11 / 1.05$ & $\mathrm{O}$ & 2 & IT(10) & PM & $\mathrm{C}$ & $\$$ & $\mathrm{P}$ \\
\hline Sample d & 46 & US & 1.72 & $1.04 / 2.40$ & $\mathrm{O}$ & 2 & $\mathrm{IT}(10)$ & PM & $\mathrm{C}$ & $\$$ & $\mathrm{P}$ \\
\hline Sample e & 63 & US & 0.47 & $-0.03 / 0.97$ & $\mathrm{O}$ & 2 & $\mathrm{IT}(10)$ & $\mathrm{PM}$ & $\mathrm{C}$ & $\$$ & $\mathrm{P}$ \\
\hline Sample f & 56 & US & 1.56 & $0.95 / 2.18$ & $\mathrm{O}$ & 2 & IT(10) & PM & $\mathrm{C}$ & $\$$ & $\mathrm{P}$ \\
\hline Sample g & 47 & US & 0.34 & $-0.24 / 0.91$ & $\mathrm{O}$ & 2 & $\mathrm{IT}(10)$ & PM & $\mathrm{C}$ & $\$$ & $\mathrm{P}$ \\
\hline Sample h & 46 & US & 1.68 & $1.01 / 2.36$ & $\mathrm{O}$ & 2 & $\mathrm{IT}(10)$ & $\mathrm{PM}$ & $\mathrm{C}$ & $\$$ & $\mathrm{P}$ \\
\hline Yamagishi (1986) & 128 & JP & 0.61 & $0.25 / 0.98$ & PGD & 4 & $\mathrm{IT}(12)$ & - & $\mathrm{C}$ & $\$$ & $\mathrm{P}$ \\
\hline Yamagishi (1988) & 196 & US & 1.16 & $0.86 / 1.47$ & PGD & 4 & $\mathrm{IT}(24)$ & - & $\mathrm{C}$ & $\$$ & $\mathrm{P}$ \\
\hline Yamagishi (1992) & 240 & JP & 2.14 & $1.36 / 2.92$ & PGD & 6 & IT(12) & - & $\mathrm{C}$ & $\$$ & $\mathrm{P}$ \\
\hline
\end{tabular}

Note. Dashes indicate that the particular characteristic is not applicable to that specific study. Study $=$ an independent coded effect size; Sample $=$ a nonindependent coded effect size; $N=$ number of participants included in the effect size estimate; $\mathrm{CO}=$ country; $\mathrm{AT}=\mathrm{Austria}$; $\mathrm{AU}=\mathrm{Australia}$; $\mathrm{BY}=$ Belarus; $\mathrm{CH}=$ Switzerland; $\mathrm{CN}=$ China; DE = Germany; DK = Denmark; GR = Greece; HK = Hong Kong; IS = Israel; IT = Italy; JP = Japan; KR = Korea; NL = the Netherlands; $\mathrm{OM}=$ Oman; RU = Russia; SA = Saudi Arabia; SE = Sweden; TR = Turkey; UA = Ukraine; UK = United Kingdom; $\mathrm{US}=$ United States; ZA $=$ South Africa; $d=$ standardized mean difference; $L L / U L=95 \%$ confidence interval with lower limit/upper limit; DV $=$ dependent variable; $\mathrm{PGD}=$ public goods dilemma; $\mathrm{PD}=$ prisoner's dilemma; $\mathrm{RD}=$ resource dilemma; $\mathrm{GS}=$ group size; IT(\#) $=$ iterated dilemma (number of iterations); $\mathrm{OS}=$ one-shot dilemma; $\mathrm{SD}=$ stranger design; $\mathrm{PM}=$ partner matching design; $\mathrm{DC}=$ decentralized punishment; $\mathrm{C}=$ centralized punishment; $\$=$ punishment is costly; $\mathrm{Fr}=$ punishment is free; $\mathrm{P}=$ paid for decisions in the dilemma; $\mathrm{NP}=$ not paid for decisions in the dilemma.

This effect size estimate could have been influenced by publication bias, as the majority of studies included in the analysis were published studies. To examine the possibility of a publication bias, we first considered the funnel plot where all studies were plotted according to their sample size and standard error. In this plot, we observed a reasonable amount of symmetrical dispersion of the effect sizes, but to examine this more formally, we used Duval and Tweedie's (2000) trim and fill approach. This method examines the symmetry of the effect sizes in the funnel plot and removes the most extreme small studies from either side of the plot while recalculating the average effect size at each iteration until symmetry is achieved. If there is publication bias, then we can expect that this approach will remove studies above the estimated overall effect size and provide an estimate of a lower estimated effect size without publication bias. Through this approach, we found that there were no studies that were trimmed above the estimated effect size. Asymmetry did exist, however, characterized by missing studies below the estimated effect size. The trim and fill approach added 11 studies above the estimated effect size and recalculated an estimated average effect size larger than the original estimated effect size $(d=0.81,95 \%$ CI $L L=0.69, U L=0.93)$. Moreover, we found a statistically significant Egger's regression intercept, intercept $=3.58, t(124)=6.29, p<.001$, which indicates bias in the data. Taken together, these results suggest that the sample did not contain a publication bias that is usually characterized by missing null findings. Instead, there seems to be a slight bias of missing studies with larger effect sizes.

As an additional measure of publication bias, we also estimated Orwin's (1983) fail-safe $N$, a statistic designed to estimate the number of unpublished findings with null results necessary to 
reduce the overall effect size to nonsignificance $(d=0.05)$. We found that Orwin's fail-safe $N$ is 1,058 . According to Hedges and Olkin (1985), to assure confidence in the results, Orwin's fail-safe $N$ should be greater than 5 times the number of studies (here, $5 \times$ $126=630)$ plus $10(630+10=640)$. Thus, we can conclude that the estimated average effect size for the punishment-cooperation relationship is robust to the presence of unpublished null results.

Rewards and cooperation. Table 2 reports the sample of studies included in this analysis that examined the rewardscooperation association $(k=33)$. Rewards had a medium-sized, positive effect on cooperation in social dilemmas $(d=0.51,95 \%$ CI $L L=0.31, U L=0.70,90 \%$ prediction interval $L L=0.27$, $U L=1.29$ ). Examining the heterogeneity of the effect size distribution indicates that there is variation in the true effect size $\left(T^{2}=\right.$
$.20, T=.45)$ and that much of this variation can be explained by between-study differences $\left(I^{2}=80.81 \%\right)$. The overall effect size distribution for these studies also contained more variation than would be expected by chance alone, $Q(24)=130.24, p<.001$.

Considering the possibility of a publication bias, we examined the funnel plot using Duval and Tweedie's (2000) trim and fill approach. This approach did indicate some asymmetry in the effect size, but this asymmetry was again the result of missing effect sizes larger than the overall estimated effect size. This approach did not remove any studies above the estimated average effect size but did remove four studies below the estimated effect size and estimated an unbiased average effect size $(d=0.64,95 \%$ CI $L L=$ $0.43, U L=0.85$ ), which was larger than the original effect size. Egger's regression intercept, however, was nonsignificant, inter-

Table 2

Studies on the Rewards-Cooperation Relationship

\begin{tabular}{|c|c|c|c|c|c|c|c|c|c|c|}
\hline Study & $N$ & $\mathrm{CO}$ & $d$ & $L L / U L$ & DV & GS & $\mathrm{IT}(\#)$ & $\mathrm{DC} / \mathrm{C}$ & $\$ / F r$ & $\mathrm{P} / \mathrm{NP}$ \\
\hline Cha (1999) & 98 & US & 1.37 & $0.93 / 1.81$ & $\mathrm{RD}$ & 8 & IT & $\mathrm{C}$ & $\mathrm{Fr}$ & $\mathrm{P}$ \\
\hline Chen, Pillutla, \& Yao (2009) & 64 & US & 0.46 & $-0.04 / 0.96$ & PGD & 4 & OS & $\mathrm{C}$ & $\mathrm{Fr}$ & NP \\
\hline Clark (2002) & 60 & $\mathrm{NZ}$ & 0.07 & $-0.44 / 0.58$ & PGD & 5 & $\mathrm{IT}(10)$ & $\mathrm{C}$ & $\$$ & $\mathrm{P}$ \\
\hline Dickinson (2001) & 80 & US & 1.04 & $0.57 / 1.51$ & PGD & 4 & $\mathrm{IT}(8)$ & $\mathrm{C}$ & $\mathrm{Fr}$ & $\mathrm{P}$ \\
\hline Sample b & 80 & US & 0.47 & $0.02 / 0.91$ & PGD & 4 & $\mathrm{IT}(8)$ & $\mathrm{C}$ & $\mathrm{Fr}$ & $\mathrm{P}$ \\
\hline Komorita \& Barth (1985) & 60 & US & 0.11 & $-0.45 / 0.67$ & PD & 3 & IT(20) & $\mathrm{C}$ & $\mathrm{Fr}$ & $\mathrm{P}$ \\
\hline Martichuski \& Bell (1991) & 48 & US & 0.66 & $0.08 / 1.24$ & RD & 3 & IT(15) & $\mathrm{C}$ & $\mathrm{Fr}$ & $\mathrm{P}$ \\
\hline McCusker \& Carnevale (1995) & 124 & US & 2.17 & $1.64 / 2.69$ & $\mathrm{RD}$ & 8 & $\mathrm{IT}(12)$ & $\mathrm{C}$ & $\$$ & $\mathrm{P}$ \\
\hline Study 2 & 48 & US & 1.88 & $1.07 / 2.67$ & $\mathrm{RD}$ & 8 & IT(12) & $\mathrm{C}$ & $\$$ & $\mathrm{P}$ \\
\hline \multicolumn{11}{|l|}{ Mulder, van Dijk, De Cremer, \& } \\
\hline Wilke (2001) & 100 & NL & 0.08 & $-0.31 / 0.47$ & PGD & 4 & OS & $\mathrm{C}$ & $\mathrm{Fr}$ & $\mathrm{P}$ \\
\hline Sample b & 38 & NL & 0.13 & $-0.51 / 0.77$ & PGD & 4 & OS & $\mathrm{C}$ & $\mathrm{Fr}$ & $\mathrm{P}$ \\
\hline Mulder (2008) & 74 & NL & -0.21 & $-0.66 / 0.24$ & PGD & 4 & OS & $\mathrm{C}$ & $\mathrm{Fr}$ & $\mathrm{P}$ \\
\hline \multicolumn{11}{|l|}{ Mulder, van Dijk, De Cremer, \& } \\
\hline Wilke (2001) & 100 & NL & 0.08 & $-0.31 / 0.47$ & PGD & 4 & OS & $\mathrm{C}$ & $\mathrm{Fr}$ & $\mathrm{P}$ \\
\hline Sample b & 38 & NL & 0.13 & $-0.51 / 0.77$ & PGD & 4 & OS & $\mathrm{C}$ & $\mathrm{Fr}$ & $\mathrm{P}$ \\
\hline \multicolumn{11}{|l|}{ Mulder, van Dijk, De Cremer, \& } \\
\hline Wilke (2006a) & 206 & NL & 0.61 & $0.17 / 1.07$ & PGD & 4 & OS & $\mathrm{C}$ & $\mathrm{Fr}$ & $\mathrm{P}$ \\
\hline Sample b & 80 & NL & 0.02 & $-0.42 / 0.45$ & PGD & 4 & OS & $\mathrm{C}$ & $\mathrm{Fr}$ & $\mathrm{P}$ \\
\hline \multicolumn{11}{|l|}{ Mulder, van Dijk, Wilke, \& De } \\
\hline Cremer (2005) & 120 & NL & 0.15 & $-0.21 / 0.51$ & PGD & 4 & OS & $\mathrm{C}$ & $\mathrm{Fr}$ & $\mathrm{P}$ \\
\hline Parks (2000) & 124 & US & 0.64 & $0.23 / 1.05$ & PGD & 4 & $\mathrm{IT}(20)$ & $\mathrm{C}$ & $\mathrm{Fr}$ & $\mathrm{P}$ \\
\hline Sample b & 124 & US & 1.17 & $0.72 / 1.62$ & PGD & 4 & IT(20) & $\mathrm{C}$ & $\mathrm{Fr}$ & $\mathrm{P}$ \\
\hline Sample c & 124 & US & 0.71 & $0.30 / 1.21$ & PGD & 4 & $\mathrm{IT}(20)$ & $\mathrm{C}$ & $\mathrm{Fr}$ & $\mathrm{P}$ \\
\hline Sample d & 124 & US & 0.40 & $-0.01 / 0.80$ & PGD & 4 & IT(20) & $\mathrm{C}$ & $\mathrm{Fr}$ & $\mathrm{P}$ \\
\hline Sample e & 124 & US & 0.29 & $-0.12 / 0.69$ & PGD & 4 & $\mathrm{IT}(20)$ & $\mathrm{C}$ & $\mathrm{Fr}$ & $\mathrm{P}$ \\
\hline Sample f & 124 & US & 0.00 & $-0.42 / 0.42$ & PGD & 4 & $\mathrm{IT}(20)$ & $\mathrm{C}$ & $\mathrm{Fr}$ & $\mathrm{P}$ \\
\hline \multicolumn{11}{|c|}{ Rand, Dreber, Ellingsen, Fudenberg, \& } \\
\hline Nowak (2009) & 54 & US & 1.41 & $0.56 / 2.27$ & PGD & 4 & $\operatorname{IT}(50)$ & $\mathrm{C}$ & $\$$ & $\mathrm{P}$ \\
\hline Rapoport \& Au (2001) & 30 & HK & 0.20 & $-0.56 / 0.96$ & $\mathrm{RD}$ & 5 & IT(90) & $\mathrm{C}$ & $\mathrm{Fr}$ & $\mathrm{P}$ \\
\hline Sefton, Shupp, \& Walker (2007) & 72 & US & 0.87 & $-0.10 / 1.83$ & PGD & 4 & IT(10) & DC & $\$$ & $\mathrm{P}$ \\
\hline Sutter, Haigner, \& Kocher (2010) & 180 & AT & 0.43 & $0.14 / 0.73$ & PGD & 4 & $\mathrm{IT}(10)$ & DC & $\$$ & $\mathrm{P}$ \\
\hline Sample b & 284 & AT & 0.82 & $0.56 / 1.08$ & PGD & 4 & $\mathrm{IT}(10)$ & DC & $\$$ & $\mathrm{P}$ \\
\hline Sutter, Linder, \& Platsch (2009) & 92 & $\mathrm{DE}$ & 0.63 & $0.40 / 0.85$ & $\mathrm{PD}$ & 2 & $\mathrm{IT}(15)$ & DC & $\$$ & $\mathrm{P}$ \\
\hline Walker \& Halloran (2004) & 48 & US & -0.17 & $-0.45 / 0.12$ & PGD & 4 & OS & DC & $\mathrm{Fr}$ & $\mathrm{P}$ \\
\hline Sample b & 48 & US & -0.11 & $-0.39 / 0.18$ & PGD & 4 & OS & DC & $\mathrm{Fr}$ & $\mathrm{P}$ \\
\hline Wit \& Wilke (1990) & 61 & NL & 0.52 & $-0.01 / 1.06$ & PGD & 10 & OS & $\mathrm{C}$ & $\mathrm{Fr}$ & NP \\
\hline Study 2 & 120 & NL & -0.43 & $-0.87 / 0.00$ & PGD & 10 & OS & $\mathrm{C}$ & $\mathrm{Fr}$ & NP \\
\hline Study 3 & 120 & NL & 0.34 & $-0.03 / 0.71$ & PGD & 10 & OS & $\mathrm{C}$ & $\mathrm{Fr}$ & NP \\
\hline Study 4 & 63 & NL & 0.47 & $-0.05 / 0.99$ & PGD & 10 & OS & $\mathrm{C}$ & $\mathrm{Fr}$ & NP \\
\hline
\end{tabular}

Note. $\quad$ Study $=$ an independent coded effect size; Sample $=$ a nonindependent coded effect size; $N=$ number of participants included in the effect size estimate; $\mathrm{CO}=$ country; $\mathrm{AT}=$ Austria; $\mathrm{DE}=$ Germany; $\mathrm{HK}=$ Hong Kong; $\mathrm{NL}=$ the Netherlands; $\mathrm{NZ}=$ New Zealand; US $=$ United States; $d=$ standardized mean difference; $L L / U L=95 \%$ confidence interval with lower limit/upper limit; DV = dependent variable; PGD = public goods dilemma; $\mathrm{PD}=$ prisoner's dilemma; $\mathrm{RD}=$ resource dilemma; $\mathrm{GS}=$ group size; $\mathrm{IT}(\#)=$ iterated dilemma (number of iterations); OS = one-shot dilemma; $\mathrm{DC}=$ decentralized punishment; $\mathrm{C}=$ centralized punishment; $\$=$ punishment is costly; $\mathrm{Fr}=$ punishment is free; $\mathrm{P}=$ paid for decisions in the dilemma; $\mathrm{NP}=$ not paid for decisions in the dilemma. 
cept $=0.03, t(24)=0.02, p=.78$. However, this later result may be due to low statistical power. The estimated fail-safe $N$ was 258 , indicating that the rewards-cooperation effect size may be robust. Overall, we conclude that our data did not contain a publication bias characterized by systematically missing studies below the average effect size. Instead, there was slight bias in the data to underestimate the effects of both rewards and punishments on cooperation.

Punishments versus rewards and cooperation. Although the punishment effect was slightly stronger than the rewards effect, comparing these two estimates using a mixed-effects analysis indicated that the effectiveness of punishments and rewards did not statistically differ in their impact on cooperation, $Q(1)=2.95, p=$ .09. Previous research has suggested that punishments may be more effective during one-shot dilemmas, whereas rewards may be more effective during iterated dilemmas. To examine these perspectives, we considered the relative effectiveness of rewards and punishments during either one-shot or iterated dilemmas. During one-shot interactions, punishments $(d=0.32,95 \%$ CI $L L=0.20$, $U L=0.43)$ did not significantly differ from rewards $(d=0.16$, $95 \%$ CI $L L=-0.03, U L=0.35), Q(1)=1.92, p=.16$, even though the punishment effect was statistically significant and the reward effect was not. During iterated dilemmas, there was no difference between punishment $(d=0.87,95 \%$ CI $L L=0.74$, $U L=0.99)$ and rewards $(d=0.81,95 \%$ CI $L L=0.55, U L=$ 1.07), $Q(1)=0.15, p=.70$.

\section{Moderators}

We now consider the possible moderators of the punishmentcooperation and rewards-cooperation effect size distributions. In the following analyses, we consider each possible moderator in turn while examining its impact on each relationship, respectively. The results of these moderator analyses on the punishmentcooperation and rewards-cooperation effect sizes are reported in Tables 3 and 4, respectively.

Cost of incentive: Costly versus free. The punishmentcooperation association was stronger when punishment was costly ( $d=0.78,95 \%$ CI $L L=0.66, U L=0.91)$ compared to when it was free $(d=0.52,95 \%$ CI $L L=0.34, U L=0.70), Q(1)=5.41$, $p=.02$. Similarly, the rewards-cooperation relationship was stronger when rewarding was costly $(d=0.97,95 \%$ CI $L L=0.57$, $U L=1.37)$ compared to when it was free $(d=0.32,95 \%$ CI $L L=$ $0.12, U L=0.52), Q(1)=8.07, p=.005$.

Table 3

Results of the Categorical Univariate Moderator Analyses on the Punishment-Cooperation Relationship

\begin{tabular}{|c|c|c|c|c|c|c|}
\hline Variable and class & $\begin{array}{l}\text { Between-class } \\
\text { effect }(Q b)\end{array}$ & $k$ & $N$ & $d$ & $T$ & $\begin{array}{c}\text { 95\% confidence interval } \\
\text { for } d \\
\text { (lower limit/upper limit) }\end{array}$ \\
\hline Costly vs. free & $5.41^{*}$ & & & & & \\
\hline Costly & & 86 & 8,264 & 0.78 & .54 & $0.66 / 0.91$ \\
\hline Free & & 39 & 3,862 & 0.52 & .52 & $0.34 / 0.71$ \\
\hline Centralized vs. decentralized & $4.31^{*}$ & & & & & \\
\hline Centralized & & 40 & 4,252 & 0.55 & .51 & $0.38 / 0.72$ \\
\hline Decentralized & & 82 & 7,647 & 0.78 & .55 & $0.65 / 0.91$ \\
\hline Experiment protocol & $24.50^{*}$ & & & & & \\
\hline Partner matching & & 48 & 3,324 & 1.02 & .60 & $0.83 / 1.21$ \\
\hline Stranger matching & & 25 & 3,320 & 0.37 & .40 & $0.20 / 0.54$ \\
\hline Iterations vs. one-shot & $41.79^{*}$ & & & & & \\
\hline Iterations with partner matching & & 74 & 5,875 & 0.92 & .57 & $0.78 / 1.07$ \\
\hline Iterations with stranger design & & 15 & 1,919 & 0.62 & .50 & $0.35 / 0.90$ \\
\hline One-shot dilemmas & & 37 & 4,396 & 0.32 & .30 & $0.20 / 0.43$ \\
\hline Type of dilemma & 1.54 & & & & & \\
\hline Public goods dilemma & & 104 & 10,147 & 0.74 & .54 & $0.62 / 0.85$ \\
\hline Resource dilemma & & 13 & 1,373 & 0.54 & .48 & $0.25 / 0.84$ \\
\hline Prisoner's dilemma & & 6 & 346 & 0.77 & .38 & $0.38 / 1.17$ \\
\hline Participant payment & $4.65^{*}$ & & & & & \\
\hline Not paid & & 12 & 1,219 & 0.31 & .63 & $-0.07 / 0.69$ \\
\hline Paid & & 114 & 10,971 & 0.74 & .52 & $0.64 / 0.85$ \\
\hline Cost-to-fine ratio & 0.61 & & & & & \\
\hline 1 to 1 & & 5 & 458 & 0.87 & .31 & $0.53 / 1.22$ \\
\hline 1 to 2 & & 7 & 644 & 0.76 & .17 & $0.55 / 0.96$ \\
\hline 1 to 3 & & 49 & 3,697 & 0.84 & .60 & $0.66 / 1.02$ \\
\hline 1 to 4 & & 4 & 405 & 0.90 & .45 & $0.41 / 1.39$ \\
\hline Country of participants & $124.59^{*}$ & & & & & \\
\hline Australia & & 5 & 568 & 1.27 & .50 & $0.79 / 1.75$ \\
\hline Israel & & 4 & 288 & 1.03 & .43 & $0.51 / 1.55$ \\
\hline Japan & & 5 & 705 & 1.07 & .51 & $0.56 / 1.57$ \\
\hline The Netherlands & & 30 & 2,761 & 0.77 & .58 & $0.54 / 1.00$ \\
\hline Russia & & 7 & 1,023 & -0.03 & .09 & $-0.12 / 0.07$ \\
\hline Switzerland & & 13 & 1,324 & 1.00 & .62 & $0.64 / 1.36$ \\
\hline United States & & 35 & 3,446 & 0.62 & .41 & $0.46 / 0.77$ \\
\hline
\end{tabular}

${ }^{*} p<.05$. 
Table 4

Results of the Categorical Univariate Moderator Analyses on the Reward-Cooperation Relationship

\begin{tabular}{|c|c|c|c|c|c|c|}
\hline Variable and class & $\begin{array}{c}\text { Between-class } \\
\text { effect }(Q b)\end{array}$ & $k$ & $n$ & $d$ & $T$ & $\begin{array}{c}\text { 95\% confidence interval } \\
\text { for } d \\
\text { (lower limit/upper limit) }\end{array}$ \\
\hline Costly vs. free & $8.07^{*}$ & & & & & \\
\hline Costly & & 8 & 992 & 0.97 & .51 & $0.57 / 1.38$ \\
\hline Free & & 18 & 1,954 & 0.32 & .35 & $0.12 / 0.52$ \\
\hline Centralized vs. decentralized & 0.01 & & & & & \\
\hline Centralized & & 19 & 2,338 & 0.50 & .53 & $0.23 / 0.77$ \\
\hline Decentralized & & 7 & 608 & 0.52 & .30 & $0.24 / 0.80$ \\
\hline Iterations & $15.47^{*}$ & & & & & \\
\hline One-shot & & 12 & 1,250 & 0.16 & .23 & $-0.03 / 0.35$ \\
\hline Iterations & & 14 & 1,656 & 0.81 & .43 & $0.55 / 1.07$ \\
\hline Type of dilemma & $7.80^{*}$ & & & & & \\
\hline Public goods dilemma & & 19 & 1,878 & 0.34 & .32 & $0.17 / 0.52$ \\
\hline Prisoner's dilemma & & 1 & 120 & 0.11 & .00 & $-0.45 / 0.67$ \\
\hline Resource dilemma & & 5 & 828 & 1.27 & .70 & $0.60 / 1.93$ \\
\hline Participant payment & $3.30^{\dagger}$ & & & & & \\
\hline Not paid & & 6 & 898 & 0.23 & .30 & $-0.08 / 0.54$ \\
\hline Paid & & 20 & 1,928 & 0.59 & .56 & $0.36 / 0.82$ \\
\hline Country & $9.78^{*}$ & & & & & \\
\hline The Netherlands & & 10 & 1,805 & 0.16 & .24 & $-0.05 / 0.37$ \\
\hline United States & & 11 & 958 & 0.88 & .61 & $0.48 / 1.28$ \\
\hline
\end{tabular}

${ }^{\dagger} p=.07 . \quad{ }^{*} p<.05$.

Incentive system: Decentralized versus centralized. The punishment-cooperation association was significantly stronger in a decentralized system $(d=0.78,95 \%$ CI $L L=0.65, U L=0.91)$ compared to a centralized system $(d=0.55,95 \%$ CI $L L=0.38$, $U L=0.72), Q(1)=4.31, p=.03$. However, regarding the rewards-cooperation effect size, there was no statistical difference between rewards being administered by a centralized $(d=0.50$, $95 \%$ CI $L L=0.23, U L=0.77)$ or a decentralized system $(d=$ $0.52,95 \%$ CI $L L=0.24, U L=0.80), Q(1)=0.02, p=.91$.

Group size. We coded the size of the group facing the dilemma and use this as continuous measure predicting the effect size in meta-regression. Group size did not moderate either the punishment-cooperation association, $b=-.01, Q(1)=0.15, p=$ .70 or the reward-cooperation association, $b=.04, Q(1)=0.28$, $p=.35$.

Type of experimental design: Partner versus stranger design. For this analysis, we included only studies that employed the Fehr and Gächter (2000) protocol. The punishmentcooperation association was stronger in the partner design $(d=$ $1.02,95 \%$ CI $L L=0.83, U L=1.21)$ than in the stranger design $(d=0.37,95 \%$ CI $L L=0.20, U L=0.54), Q(1)=25.50, p<$ .001 .

One-shot versus iterated dilemmas. In some experiments on punishment, researchers have participants play a social dilemma repeatedly, but during each round, they are randomly assigned with a new group of participants in the dilemma (the stranger design). When testing the effect of iterations on the punishmentcooperation effect size, we considered the difference among three conditions: (a) iterations with a partner matching design, (b) iterations with a stranger design, and (c) one-shot interactions. However, for the rewards-cooperation relationship, there were no studies that combined iterations with a stranger design, and therefore, we can compare iterations only of a partner matching design and one-shot interactions.
The punishment-cooperation association was stronger in the iterated dilemmas with partner matching $(d=0.92,95 \%$ CI $L L=$ $0.78, U L=1.06)$ compared to the iterated dilemmas with a stranger design $(d=0.62,95 \% \mathrm{CI} L L=0.35, U L=0.90)$ and the one-shot dilemmas $(d=0.32,95 \%$ CI $L L=0.21, U L=0.42)$, $Q(2)=44.42, p<.02$. Similarly, the rewards-cooperation association was stronger during iterated dilemmas $(d=0.76,95 \% \mathrm{CI}$ $L L=0.50, U L=1.03)$ than in the one-shot dilemmas $(d=0.16$, 95\% CI $L L=-0.03, U L=0.35), Q(1)=13.03, p<.001$.

We examined whether the incentives-cooperation association would become stronger with an increase in the number of iterations of the dilemma. In a subsequent analysis, we use the number of iterations as a continuous variable predicting the effect size in meta-regression. Iterations were nonsignificantly related to the effect size distribution for both punishment, $b=.005, Q(1)=$ $3.38, p=.06$, and rewards, $b=.003, Q(1)=1.10, p=.29$. However, this random-effects model may be conservative, especially with a small sample of studies. Using a fixed-effects model, there was a significant positive effect of iterations on the rewardscooperation effect size, $b=.009, Q(1)=6.60, p=.01$.

Type of dilemma. There are three main types of dilemmas represented in our analysis: public goods dilemmas, prisoner's dilemmas, and resource dilemmas. The punishment-cooperation association did not differ across the type of dilemma, $Q(2)=1.52$, $p=.46$. Specifically, this effect size did not statistically differ between the prisoner's dilemma $(d=0.54,95 \%$ CI $L L=0.25$, $U L=0.84)$, the public goods dilemma $(d=0.74,95 \%$ CI $L L=$ $0.62, U L=0.85)$, and the resource dilemma $(d=0.77,95 \% \mathrm{CI}$ $L L=0.38, U L=1.17)$.

However, for the rewards-cooperation association, there was a significant difference between the types of dilemmas, $Q(2)=7.80$, $p=.02$. The rewards-cooperation linkage was the strongest in the resource dilemma $(d=1.27,95 \%$ CI $L L=0.60, U L=1.94)$ compared to the public goods dilemma $(d=0.34,95 \%$ CI $L L=$ 
$0.17, U L=0.52)$ and the prisoner's dilemma $(d=0.11,95 \% \mathrm{CI}$ $L L=-0.45, U L=0.67)$.

Participant payment: Paid versus not paid. Punishment was more effective when participants were paid for the outcomes of the dilemma ( $d=0.75,95 \%$ CI $L L=0.64, U L=0.85$ ) relative to when they were making decisions about hypothetical amounts of money $(d=0.31,95 \% \mathrm{CI} L L=-0.07, U L=0.69), Q(1)=$ $4.65, p=.03$. The rewards-cooperation association was marginally significantly larger when participants were paid for their decisions ( $d=0.59,95 \%$ CI $L L=0.36, U L=0.82)$ than not paid for their decisions in the dilemma $(d=0.23,95 \%$ CI $L L=-0.08$, $U L=0.54), Q(1)=3.30, p=.07$.

Cost-to-fine ratio in punishment studies. We found that the cost-to-fine ratio did not moderate the punishment-cooperation effect size, $Q(3)=0.61, p=.89$. Specifically, we did not observe a statistical difference in the estimated punishment-cooperation effect size between conditions when one monetary unit purchased a reduction in one $(d=0.87)$, two $(d=0.76)$, three $(d=0.84)$, or four $(d=0.90)$ monetary units of the person punished.

Country of participants. When analyzing the effect of punishment on cooperation across countries we considered those countries represented by four or more effect sizes. We found that the impact of punishment on cooperation varied significantly across countries, $Q(7)=124.61, p<.001$. The punishmentcooperation effect size was strongest in studies from Australia $(d=1.27)$, Japan $(d=1.07)$, Israel $(d=1.03)$, Switzerland $(d=$ $1.00)$, the Netherlands $(d=0.77)$, and the United States $(d=0$ .62). However, punishment had no effect on cooperation in studies conducted in Russia $(d=-0.03)$.

The rewards-cooperation effect size sample contained much less diversity in countries. We found that rewards were more effective at enhancing cooperation in the United States $(d=0.88)$ than in the Netherlands $(d=0.16), Q(1)=9.78, p=.002$.

\section{Multiple Regression Model}

Some moderators are intercorrelated-for example, there was an exceptionally strong correlation between cost of incentives and whether the incentive was centralized or decentralized $(r=.83$, $p<.001)$. To examine such overlapping moderators, we conducted a random-effects multiple regression analysis using method of moments estimations with SPSS macros provided by Lipsey and Wilson (2001). In this analysis, we combined both punishment and reward effect sizes as the dependent variable and included seven predictors of the effect size: (a) type of incentive (punishment vs. reward), (b) cost of incentive (free vs. costly), (c) source of incentive (centralized vs. decentralized), (d) number of iterations, (e) participant payment (paid vs. not paid), (f) group size, and (g) whether the study was conducted by either an economist or psychologist. The model predicted a significant amount of variance in the effect size distribution, $R^{2}=.11, Q(7)=22.90, p=001$. Supporting earlier analyses, we found that three variables moderated the effect size: cost of incentive $(\beta=.26, p=.04)$, participant payment $(\beta=.21, p=.02)$, and iterations $(\beta=.12, p=.08)$. After controlling for the variance explained by these other variables, source of incentives did not moderate the effect size. ${ }^{4}$ Similarly, there was no effect of type of incentive, group size, or whether studies were conducted by economists versus psychologists.

\section{Discussion}

Using an interdependence-theoretic framework, we proposed that rewards and punishment should promote cooperation and identified two variables - cost of incentives and source of incentives - that might influence the effectiveness of these incentives in promoting cooperative behavior. A meta-analysis involving 187 effect sizes revealed that reward and punishment exhibit a substantial and statistically equivalent positive effect on cooperation. ${ }^{5}$ Thus, the main finding is that incentives reliably enhance cooperative behavior. Furthermore, we found that the effectiveness of these incentives is stronger when the incentives are costly to administer. We suggested earlier that the administration of costly incentives might signal a strong commitment to the goal of promoting cooperation, rather than simply pursuing self-interest. It is the perceptions of these benevolent interpersonal motives that may explain why incentives are more effective to the degree that they are more costly to administer.

We also believed that incentives that were decentralized (i.e., provided by actual participants in the cooperative venture) would induce more cooperation than centralized incentives (i.e., those provided by external sources). Whereas the meta-analysis provided support for this hypothesis, after statistically controlling for cost, centralized and decentralized incentive systems did not significantly differ in their impact on cooperation. We also found that punishments were more effective during iterated dilemmas when participants continued to interact in the same group, compared to both iterated dilemmas with reassignment to a new group after each trial and one-shot dilemmas. The incentive-cooperation effect size differed across countries but was found to be unrelated to other study characteristics (e.g., group size or the cost-to-fine ratio).

In what follows, we evaluate the evidence relevant to the hypotheses of interdependence theory, discuss some intriguing issues raised by the meta-analysis, and suggest directions for future research. We start with the first question raised in this article.

\footnotetext{
${ }^{4}$ We also conducted a random-effects multiple regression model including the cost of incentives and source of incentives as predictors of the effect size. Again, cost of incentives was the only significant predictor of the effect size in this model.

${ }^{5} \mathrm{We}$ are the first, to our knowledge, to conduct a comprehensive meta-analytic review of the effect of incentives on cooperation. At the same time, we should acknowledge an earlier meta-analysis, conducted by Zelmer (2003), which reviewed factors affecting cooperation in public good settings. This meta-analysis provided more preliminary evidence, in that the analysis was smaller in scope (27 studies) and included only a few studies in which punishment was manipulated. Moreover, nearly all studies included were conducted by behavioral economists, and no moderators of the effect sizes were examined. The present meta-analysis complements this past work especially by including a much broader sample of studies from both economics and psychology that enabled us to test several theoretically interesting moderators. Moreover, the present meta-analysis complements recent narrative reviews of incentives and cooperation in both economics (Chaudhuri, 2011) and psychology (Shinada \& Yamagishi, 2008).
} 


\section{Are Rewards and Punishment Effective Promoters of Cooperation?}

A great deal of research has focused on the role of incentives as tools for promoting cooperation in social dilemmas. Incentives can be seen as structural solutions to resolving social dilemmas in that they affect the interdependence structure of the social dilemma by reducing the conflicts of interest (e.g., Kollock, 1998; Van Lange \& Joireman, 2008; Yamagishi, 1986, 1988). We note immediately that some investigators have proposed that incentives may energize a set of interrelated psychological processes that might reduce cooperation in social dilemmas, including (a) decreasing intrinsic motivation for cooperation; (b) undermining feelings of autonomy, control, and freedom; and (c) fueling psychological reactance. Thus, a key question drove our investigation: Given that research is generally supportive of these psychological mechanisms related to hidden costs of incentives (Lepper \& Greene, 1978), can incentives effectively increase cooperation?

In support of Hypothesis 1, the present findings provide unequivocal evidence that rewards and punishment are effective promoters of cooperation in social dilemmas, explaining approximately $3 \%-12 \%$ of the variance in cooperation. We posit that incentives influence cooperation in a structural manner, such that the conflicts among group members are reduced. In line with interdependence theory (and game theory), people are more likely to cooperate to the degree that the incentives of collective interests are enhanced and the incentives for self-interest are reduced (Andenaes, 1971; Bankston \& Cramer, 1974; Komorita \& Parks, 1995; Rapoport \& Chammah, 1965; Zimring, 1971). As such, the first contribution of the present meta-analysis is that, although incentives might undermine intrinsic motivation and even cooperation in several important ways (e.g., Lepper \& Greene, 1978), the costs of these undermining mechanisms are outweighed by incentives reducing the conflicts of interest. In other words, reward and punishments effectively increase cooperation.

\section{What Factors Promote the Effectiveness of Reward and Punishment?}

Interdependence theory is highly useful in understanding how social circumstances might magnify the effectiveness of incentives. In particular, interdependence theory emphasizes the interpersonal motives of a person as well as the interpersonal motives that a person perceives in others. Indeed, we have suggested that rewards and punishment are effective when they are associated with the pursuit of collective interest, rather than self-interest. In fact, the decision to provide incentives can be viewed as a secondorder social dilemma: People in the dilemma can either free ride on others' provision of incentives for cooperation or sacrifice selfinterest to provide incentives for collective benefit. Indeed, modern economic theories and research have provided evidence that inequality aversion can predict sacrifice for collective interest in terms of both cooperation (Fehr \& Schmidt, 1999) and the propensity to punish noncooperators (e.g., Fowler, Johnson, \& Smirnov, 2005). Although delivering incentives typically involves the sacrifice of self-interest for collective benefit, this does not necessarily mean that others will always perceive that incentives are directed toward collective benefit and the question becomes, What situations enable perceptions that incentives are being administered in the pursuit of collective interest?
Cost of incentive. The meta-analysis provides good support for Hypothesis 2, the prediction that incentives would be more effective when the incentives were costly to administer compared to when incentives were free. We reasoned from interdependence theory that when individuals receive incentives involving cost to the provider, they are more likely to perceive that the provider is relatively more concerned about the collective, relative to when incentives are free. In fact, Fehr and Gächter (2002) labeled costly punishment altruistic because they demonstrated that people use costly punishment in the absence of immediate, material benefits for the self or their current group. If individuals perceive that others provide costly incentives to cooperate as a result of cooperative intentions, this may affect other downstream psychological processes, such as the formation of expectations of subsequent behavior, development of social norms, and formation of positive reputations, all of which may increase cooperation.

Recent research has suggested complementary (and alternative) explanations of why cost of incentives moderates their impact on cooperation. Several studies have shown a so-called price effect of punishment, such that people punish more when it costs less (C. M. Anderson \& Putterman, 2006; Andreoni, Harbaugh, \& Versterlund, 2003; Carpenter, 2007a; Egas \& Riedl, 2008; Ostrom et al., 1992). A possible implication of more frequent costless incentives is that they heighten the chances of receiving incentives and thus may increase cooperation. However, this perspective would predict a stronger incentive-cooperation linkage under conditions of costless sanctions - which is at odds with the results of this metaanalysis.

Another implication of the price effect, however, is that low incentive costs may evoke more antisocial punishment (e.g., punishment of cooperators by defectors; Cinyabuguma, Page, \& Putterman, 2005; Falk, Fehr, \& Fischbacher, 2005; Herrmann et al., 2008), whereas high incentive costs may make people more thoughtful of when and to whom to provide incentives, leading to more well-considered incentive choices. More specifically, costly rewards (punishments) will more selectively be delivered to cooperators (defectors). Thus, under conditions of costly incentives, perceived fairness of the incentives will be enhanced, and a norm of cooperation may be communicated more clearly. Alternatively, a costly incentive from a fellow participant in a dilemma may carry additional social reinforcement of the desired behavior, relative to an equivalent incentive that is free (Masclet et al., 2003; Noussair $\&$ Tucker, 2005). Future research should further examine if perceived cooperative intent motives mediate the effect of costly incentives on cooperation, while controlling for alternative explanations, such as the frequency of incentives, fairness of incentives, communication of norms, and social reinforcement.

Source of incentive. In our sample of studies, source of incentives was strongly correlated with the cost of incentives. Past research has mostly used paradigms that involve either decentralized and costly incentives or centralized and free incentives. When we controlled for the variance in the effect size accounted for by cost of incentives, source of incentives did not account for a significant amount of variance in the effect size. Hence, Hypothesis 3 was not supported.

One explanation is that centralized sanctioning systems may actually increase cooperation more than decentralized sanctioning systems. Earlier, we reasoned that centralized systems may be less effective than decentralized systems because (a) an authority who 
administers incentives can be regarded as powerful, (b) people often associate power with self-interest motives (compared to similar status others), and (c) people tend to have negative reactions to power asymmetries. At the same time, people are often more likely to be influenced by more, compared to less, powerful persons. Traditionally, power is defined in terms of the ability to move others through a wide range of outcomes (Thibaut \& Kelley, 1959), and the abilities to reward others and to punish others form two important bases of power and influence (French \& Raven, 1959). Complementing these classic conceptualizations, more recent research has revealed that feelings of power tend to reinforce the influence that power affords (C. Anderson, Keltner, \& John, 2003). For example, in negotiations, low-power individuals concede more to high-power individuals than the other way around (Butt \& Choi, 2010; De Dreu \& van Kleef, 2004; Overbeck, Neale, \& Govan, 2010; Sinaceur \& Tiedens, 2006; van Kleef, De Dreu, \& Manstead, 2004). Also, when groups are unsuccessful in managing a public good (Messick et al., 1983; Rutte \& Wilke, 1984), then group members see the installation of a leader as a fruitful way to solve the free-rider problem (Samuelson, 1991; Yamagishi, 1986).

In addition, the coding of source of incentives may reflect not only a difference in power or centralization but also a difference of personal involvement in the social dilemma. After all, an authority (e.g., the experimenter) may be less involved in the social dilemma itself than a typical group member (e.g., a participant). Thus, our reasoning that the source of incentives reflects power differentials in the dilemma may be confounded with personal involvement. This consideration is important because, in decentralized systems, a fellow group member may promote various feelings, such as common fate ("we are in this together") or identity (i.e., he or she is "one of us") that might promote trust (Brewer \& Kramer, 1986). In contrast, feelings of common fate or shared identity with authorities may be considerably weaker because the personal involvement of authorities is weaker than that of the prototypical group member (Hogg \& Terry, 2000). Thus, it is plausible that, along with differences in power, feelings of common fate and shared identity help people understand psychological consequences of centralized and decentralized incentive systems. We encourage investigators to manipulate power or centralization while keeping personal involvement constant. Such research not only could systematically test how power and centralization affect the extent to which incentive systems influence cooperation but also could inform the underlying processes, such as the undermining of trust in others, perceived cooperative motives, or perceived fairness of incentives.

Insofar as there was an effect of source of incentive, this effect disappeared, while the effect of cost of incentive remained significant. One might speculate that the perceived cost of incentives might to some degree override the potential effect of source of incentives. What matters most is not who administers the incentives but the intentions that are perceived to underlie the administration of incentives. Genuine intentions to safeguard and promote collective interests, rather than self-interest, are seemingly more persuasive when such incentives are costly. This finding informs us about the importance of designing studies where cost of incentives and source of incentives are orthogonally manipulated. The results of such studies will inform theory and may provide relatively concrete guidelines as to how to effectively administer rewards and punishments in social dilemmas.

\section{Are Rewards and Punishment Equally Effective?}

Earlier research is inconclusive on this topic. Although some studies have reported that rewards are more effective than punishment (Komorita \& Barth, 1985; McCusker \& Carnevale, 1995), other studies have suggested that punishment is more effective than reward (Andreoni et al., 2003; Rapoport \& Au, 2001; Stern, 1976) or that there is no difference between the two incentives in terms of effectiveness (Rand et al., 2009; Sefton, Shupp, \& Walker, 2007). The results of the meta-analysis suggest that rewards and punishment have equivalent main effects on cooperation. Although there is a slightly larger effect size estimate of punishment $(d=0.70)$ than rewards $(d=0.51)$, this is only marginally significant, and the analysis of bias in our sample suggests the rewards-cooperation effect size may be underestimated. Therefore, there is no strong empirical reason to conclude that reward and punishments differ in their overall effectiveness as motivators of cooperation in social dilemmas.

Previous theory and research have suggested that punishments should exert a quicker effect on behavior compared to rewards (Azrin \& Holz, 1966; Driscoll, 2005; Gershoff, 2002; Skinner, 1953). Conversely, rewards have been considered to result in better long-term learning than punishments (Driscoll, 2005; Gershoff, 2002; Holz \& Azrin, 1962; Skinner, 1953). We analyzed the differences between reward and punishment in both one-shot and iterated dilemmas. In one-shot dilemmas, rewards had a small marginally significant positive effect on cooperation $(d=0.16)$, whereas punishments had a slightly larger significant positive effect on cooperation ( $d=0.32$ ), although these differences were not statistically significant. Similarly, there was no significant difference between rewards and punishment during iterated dilemmas. Therefore, in the context of social dilemmas, there is no difference between rewards and punishment as either short-term or long-term promoters of cooperation.

Although the present findings provide strong evidence that rewards and punishments do not substantially differ in their impact on cooperation, it may still be that these incentives differentially affect either expectations of cooperation, intrinsic motivation, or ethical concerns during social dilemmas. More insight into these questions may lead to the conclusion that, despite the fact that rewards and punishments increase cooperation to an equal extent, it nevertheless matters which incentive system is installed. This is especially true in situations in which cooperation levels depend, at least in part, on individuals' willingness to cooperate or situations in which there might be some uncertainty regarding the actual administration of the incentive (e.g., when it is not easy to catch the noncooperators). Under these conditions, rewards or punishments may be more or less effective depending on their indirect effect on individuals' thoughts, feelings, and motivations in social dilemmas.

\section{Emerging Issues for Future Study: The Psychology and Economics of Incentives}

One benefit of meta-analytic reviews is that they identify areas of empirical investigation that are especially promising. Moreover, the current meta-analysis includes studies from different disciplines, which gives rise to some intriguing issues relevant to all disciplines that study human cooperation. We now discuss the 
issues of (a) incentives over time, (b) effectiveness versus efficiency of incentives, (c) participant payment, (d) and the importance of culture.

Incentives over time: Beyond reinforcement and punishment. Interestingly, we found that incentives, both reward and punishments, more strongly increased cooperation during iterated dilemmas than in one-shot dilemmas and that this association became even stronger with increased iterations. So, incentives became more effective over time. At first glance, this result highlights the role of incentives in learning cooperation. After all, learning theory suggests that, over time, rewards (punishments) will increase (decrease) the probability of subsequent sanctioned behavior (Skinner, 1953). In such situations, learning and performance are enhanced over time through patterns of positive task feedback on performance.

However, the finding that incentives become more effective over time is also in line with an interdependence-theoretical perspective. In social dilemmas, incentives generally encourage not only one's motivation to behave cooperatively (i.e., one's own motives) but also one's expectations about others' cooperation (i.e., believed or perceived others' motives). For example, prosocial motives to cooperate may not affect cooperation unless the perceivers expect others to cooperate (see Pruitt \& Kimmel, 1977), and expectations are strongly influenced by past behavior (e.g., Van Lange, 1999; Van Lange, Ouwerkerk, \& Tazelaar, 2002). Therefore, incentives may be more effective after several trials because people have developed perceived cooperative motives via group members' sanctioning behavior, generating expectations of future cooperation. Similarly, the activation of reputational concerns, the development of social norms prescribing cooperation, and the weakening of potentially undermining processes (such as feelings of injustice, feelings of being gypped; see Kerr et al., 2009; Mulder et al., 2006a; Nowak \& Sigmund, 2005; Tenbrunsel \& Messick, 1999; Yamagishi, 1986) may each unfold over time, thereby accounting for longer term rather than immediate effectiveness of incentives. Also, the emotions that can be evoked by rewards (e.g., joy, gratitude, and pride) and punishment (e.g., anger, guilt, and shame) might affect the duration of their impact by promoting a set of positive versus negative cycles of social interaction (e.g., Keltner, van Kleef, Chen, \& Kraus, 2008). Although such issues are speculative at the moment, they are important as future research may help people to understand the psychological processes that underlie the impact of incentives on cooperation over time.

As both individual learning and group processes may explain the differential effects in one-shot and iterated dilemmas, we also compared the effectiveness of incentives in a stranger design to both one-shot and iterated dilemmas using the partner matching design. Recall that, in the stranger design, individuals play a dilemma repeatedly but, for each trial, are randomly assigned to a new group of partners. If learning is the primary mechanism responsible for incentives' greater effectiveness during iterated dilemmas, then one could expect that (a) the stranger design would have an equally effective impact on the punishment-cooperation association, compared to iterated dilemmas with a partner matching design, and (b) both the stranger and the partner matching designs would show stronger punishment-cooperation effects than one-shot dilemmas. Yet this is not what we found. In fact, the partner matching studies with iterations had a larger punishment- cooperation effect size compared to the stranger design studies with iterations, which had a larger effect than one-shot dilemma studies. These findings suggest that the effect of punishment during iterated dilemmas is not only due to learning principles but also due to processes related to group norms, generating expectations of group cooperation, and strategically developing reputations (e.g., Fehr \& Fischbacher, 2004; Fehr, Fischbacher, \& Gächter, 2002). Whether this is also the case for rewards remains unclear as we did not find studies in which the stranger protocol was employed while studying the impact of rewards on cooperation.

Paid versus hypothetical dilemmas. We were particularly intrigued by the finding that both rewards and punishments were more effective when participants were actually paid for their decisions rather than making hypothetical decisions without monetary consequences. Similarly, Yamagishi (1988) found that when cooperation was more costly for individuals, then punishment was more likely to result in higher levels of cooperation. Two simple messages emerge from the current findings. First, the effect of costliness of cooperation on the punishment-cooperation association generalizes to the rewards-cooperation association. Second, researchers interested in testing theory of the incentives-cooperation linkage should test these hypotheses under conditions of costly cooperation.

Moreover, it is interesting that disciplines differ in the norms for paying participants (or not). For example, in psychology, it is not uncommon to examine social dilemmas involving hypothetical outcomes, whereas, in (behavioral) economics, there are stronger norms to examine social dilemmas involving actual cash. The present findings suggest the possibility that incentives may matter more when the stakes are greater (as for real money). This possibility, could open a new line of research because some social dilemma outcomes are indeed material, but many social dilemmas also involve immaterial outcomes (e.g., contributing to a positive group spirit) or intermediate ones, which sometimes are quite easy to translate into actual cash (e.g., time and effort). Thus, the current results suggest that incentives are more effective in conditions with concrete material outcomes or when outcomes are framed according to these material benefits of cooperation. At the same time, future research may consider whether incentives are indeed relatively less effective during dilemmas involving immaterial outcomes and, if so, how the effectiveness of incentives under these conditions can be enhanced.

Incentives and group size. Group size did not moderate the effect of incentives on cooperation. ${ }^{6}$ Yamagishi (1992) suggested that group size would increase the magnitude of the incentive-

\footnotetext{
${ }^{6}$ Casari (2005) argued that any difference between punishment behaviors in larger, compared to smaller, groups may result from different punishment protocol used in experiments. Specifically, during decentralized punishment protocol, researchers either allow a fixed or variable fine-to-fee ratio. In short, Casari noticed that the variable fine-to-fee ratio used in these studies creates a condition where it is cheaper to punish defectors in larger, compared to smaller, groups, and so, people are more likely to punish in larger groups. We examined the effect of group size on the punishment-cooperation relationship in 14 studies that used a fixed fine-to-fee ratio. We did not find that group size moderated the effect size. We did not have enough studies that used a variable fine-to-fee ratio to test for the association under this condition.
} 
cooperation relationship because people are more likely to expect others to pursue immediate self-interest and support the incentive system. Although there is evidence supporting this line of reasoning, such studies were not included in the meta-analysis because they did not examine effects of rewards and punishment on cooperation and typically involved relatively large group sizes (e.g., Kerr, 1989). In contrast, our meta-analysis included only studies on rewards and punishment, and that body of research typically includes small group sizes (where the modal of group size was four persons). Thus, it is still possible that group size moderates the effectiveness of punishment when group size is observed across a more extensive range. To date, relevant studies have compared relatively small groups (e.g., five- vs. 10-person groups: Carpenter, 2007b; Carpenter, Bowles, Gintis, \& Hwang, 2009; four- vs. eight-person groups: Yamagishi, 1992), and the results are inconclusive.

One might speculate that the psychological effects of group size become especially pronounced if one moves beyond at least five members. For example, according to the core configurations of group size, one should move beyond the five-person group to understand the unique adaptive challenges posed by larger groups (Caporael, 1997). Compared to relatively small groups (roughly seven members or fewer), which are often challenged by concrete forms of cooperation (often face-to-face interactions, such as foraging, or working on a common task), larger groups (e.g., 30 members or more) feature forms of exchanges (e.g., migration, environmental behavior, or information exchange) that are more global, abstract, and less strongly rooted in social interactions yielding concrete team products (Brewer \& Caporael, 2006; Caporael, 1997). We suggest that one important function of a metaanalytic review is that it identifies gaps in the literature or domains that have not been fully examined. As such, the present research suggests that the study of larger groups may provide important answers to the impact of group size on the use and effectiveness of rewards and punishment in social dilemmas.

Effectiveness versus efficiency. As noted earlier, the current meta-analysis leaves little doubt about the effectiveness of rewards and punishment. A next, more challenging question is whether these incentives are efficient. Do the material gains for the collective outweigh the sum of the material costs of the incentives that are administered? Indeed, this question relates to the issue of group efficiency, which has recently been addressed in prior research on punishment in particular. It appears that group efficiency is undermined shortly after the administration of punishments (e.g., Dreber, Rand, Fudenberg, \& Nowak, 2008; Egas \& Riedl, 2008). In particular, there is a reduction in group efficiency with only a few iterations of the dilemma (e.g., 10 iterations or fewer). In these dilemmas, when incentives are added, there is an initial decrease in group payoffs, but over iterations, an increase in such payoffs occurs (e.g., Fehr \& Gächter, 2000; Gächter et al., 2008; Nikiforakis \& Normann, 2008).

Although various mechanisms are possible, one is that early (and frequent) punishment of noncooperators encourages cooperation, which reduces the need for punishment on subsequent trials. There is also evidence that the potential for reputation monitoring (Rockenbach \& Milinski, 2006), for coordination in the provision of incentives (Boyd, Gintis, \& Bowles, 2010), and for self-chosen punishment systems (vs. enforced punishment systems; Gürerk, Irlenbusch, \& Rockenbach, 2006) can magnify the efficiency of incentives. Thus, there is tentative evidence suggesting that incentives are efficient, at least over time, and that several contextual variables need to be examined to understand when and why they are especially efficient. Perhaps the most intriguing dilemma is the conflict between two pressures: (a) Incentives that are more costly logically undermine efficiency, and (b) incentives that are more costly are more effective at increasing cooperation and so may bring about more benefits at the collective level.

The importance of culture. Building on earlier advances (Henrich et al., 2001), recent research has started to examine how features of the situation vary in their impact on cooperation across different cultures (e.g., Herrmann et al., 2008). The findings of the present meta-analysis, like those of Herrmann et al. (2008), reveal that the impact of incentives on cooperation varies significantly between countries. To illustrate, in Russian samples (Gächter \& Herrmann, 2011; Herrmann et al., 2008), decentralized incentives tend to have no impact on cooperation $(d=-0.03)$ and reduce group profits. In many other countries, however, punishment has a positive impact on cooperation (e.g., United States $d=0.62$ ) and enhances group efficiency. Herrmann and colleagues, in an exceptionally extensive cross-cultural study, found that countries varied substantially in their tendency to engage in antisocial (or perverse) punishment, that is, the punishment of cooperators by defectors. Among the more plausible accounts of these cultural differences is the notion that cultures differ in social norms about how to interact with strangers-people outside of informal networks that are studied in these experiments (Gintis, 2008). For example, cultures may differ in terms of how much trust they have in strangers (especially their generosity) and the desire to communicate the norm to not be excessively generous with strangers.

Setting aside specific explanations, we regard these findings as important because if we did not have such an extensive cross-cultural database, we might have been tempted to generalize our results beyond our largely Western sample (Herrmann et al., 2008). For example, the fact that costly incentives enhance cooperation, compared to costless incentives, may not extend to the Russian sample. Given the limited number of studies in several other countries, we were unable to examine the effect of moderators in a sample of each country. For example, certain countries may more or less benefit from a centralized, opposed to decentralized, incentive system. Examining such questions will undoubtedly make an important contribution. Additionally, future research on culture and cooperation should move beyond simply comparing samples from different countries (see Gächter, Herrmann, \& Thöni, 2010). Instead, this work may profit by comparing samples from different groups of countries that have different cultural backgrounds (e.g., ex-communist vs. advanced industrial societies; Inglehart \& Baker, 2000). This approach may more effectively identify dimensions of culture that affect the impact of incentives on cooperation in social dilemmas.

\section{Strengths and Limitations}

We should acknowledge at least three limitations of the present research. First, psychologists and economists have employed different paradigms in manipulating incentives. For example, whereas economists have traditionally used a decentralized system of punishment, psychologists have used a cen- 
tralized system of punishment. It may be that some subtle differences in experimental protocol between economists and psychologists may explain the differences observed between certain moderators coded along this divide. Yet we are not overly concerned about this limitation. In fact, we coded whether the studies were conducted by either an economist or psychologist and controlled for this variable in a multiple regression model predicting the effect size. This factor was not a significant moderator. Intriguingly, there is recent evidence suggesting that participants in experiments conducted by psychologists versus economists may differ in their interpersonal orientations: Prosocial orientation (i.e., the tendency to enhance equality in outcomes and joint outcomes) is the dominant orientation among psychology students, whereas individualistic orientation (i.e., the tendency to enhance outcomes for self with no or very little regard for others' outcomes) is the dominant orientation among economics students (see Van Lange, Schippers, \& Balliet, in press). Such evidence is potentially important to understanding how participants might respond to external forces that seek to promote cooperation.

Second, there are fewer studies on rewards than punishments, reducing statistical power to detect moderators of the rewardscooperation association. This is a key area for further investigation.

Third, some studies were not included in this analysis because they lacked the required statistical information to calculate the effect size. Accordingly, we employed a mixed-effects analysis, assuming we did not have all studies in the population of studies represented in our sample. A mixed-effects analysis is a more conservative test of moderator effects and should provide more confidence that the results will generalize across the population of studies.

\section{Concluding Remarks}

Reward and punishment, as two distinct incentives, are classic concepts with a long history in psychology. They are central in the basics of psychology, providing the conceptual foundation for behaviorism and the study of performance and learning. Even though incentives may provide hidden costs, such as reduced intrinsic motivation to cooperate, the results of this meta-analysis support the position of Hobbes (1651) and Hardin (1968): Incentives do promote cooperation in social dilemmas. More interesting, however, is that a generalized learning mechanism thought to underlie the effect of incentives on performance and learning may not be an all-encompassing explanation of the effect of incentives on cooperation with interdependent others. Punishment was most effective when individuals interacted with the same group over several trials, compared to when individuals were reassigned to a new group after each trial. Therefore, several other more explicitly social mechanisms may underlie the effectiveness of incentives, such as reputation, the communication of social norms, and the perceived motives of others in the dilemma.

Aligned with this perspective, interdependence theory provides an insightful analysis that helps one understand how the effectiveness of incentives can be further promoted. This analysis links an individual's psychological processes to the broader interpersonal circumstances in which behavioral patterns evolve and are reinforced. Incentives are more effective when incentives are costly. Not only is this finding of great theoretical importance, it may be essential to the successful functioning of teams, schools, and organizations. After all, these groups are all faced with the basic question, How can one promote cooperative behavior among group members? Whether it makes human beings a sorry lot or not, rewards and punishments do matter (just as do hopes and fears). Knowing that certain individuals provide incentives with the noble intent to advance collective interests, even at a cost to themselves, seems a source of inspiration, rather than a source of frustration.

\section{References}

References marked with an asterisk indicate studies included in the meta-analysis.

Andenaes, J. (1971). Deterrence and specific offenses. University of Chicago Law Review, 38, 37-53. doi:10.2307/1599029

Anderson, C., Keltner, D., \& John, O. P. (2003). Emotional convergence between people over time. Journal of Personality and Social Psychology, 84, 1054-1068. doi:10.1037/0022-3514.84.5.1054

Anderson, C. M., \& Putterman, L. (2006). Do non-strategic sanctions obey the law of demand? The demand for punishment in the voluntary contribution mechanism. Games and Economic Behavior, 54, 1-24. doi:10.1016/j.geb.2004.08.007

Andreoni, J., Harbaugh, W., \& Versterlund, L. (2003). The carrot or the stick: Rewards, punishments, and cooperation. American Economic Review, 93, 893-902. doi:10.1257/000282803322157142

Azrin, N. H., \& Holz, W. C. (1966). Punishment. In W. A. Honig (Ed.), Operant behavior: Areas of research and application (pp. 380-447). New York, NY: Appleton-Century-Crofts.

Bankston, W. B., \& Cramer, J. A. (1974). Toward a macro-sociological interpretation of general deterrence. Criminology: An Interdisciplinary Journal, 12, 251-280. doi:10.1111/j.1745-9125.1974.tb00635.x

*Bell, P. A., Petersen, T. R., \& Hautaluoma, J. E. (1989). The effect of punishment probability on overconsumption and stealing in a simulated commons. Journal of Applied Social Psychology, 19, 1483-1495. doi: 10.1111/j.1559-1816.1989.tb01460.x

*Bochet, O., Page, T., \& Putterman, L. (2006). Communication and punishment in voluntary contribution experiments. Journal of Economic Behavior \& Organization, 60, 11-26. doi:10.1016/j.jebo.2003.06.006

*Bornstein, G., \& Weisel, O. (2010a). Punishment, cooperation, and cheater detection in "noisy" social exchange. Games, 1, 18-33. doi: 10.3390/g1010018

*Bornstein, G., \& Weisel, O. (2010b). [A replication of the study reported in "Punishment, Cooperation, and Cheater Detection in 'Noisy' Social Exchange"]. Unpublished raw data.

Boyd, R., Gintis, H., \& Bowles, S. (2010, April 30). Coordinated punishment of defectors sustains cooperation and can proliferate when rare. Science, 328, 617-620. doi:10.1126/science.1183665

Brehm, J. W. (1966). A Theory of psychological reactance. Oxford, England: Academic Press.

Brewer, M. B., \& Caporael, L. R. (2006). An evolutionary perspective on social identity: Revisiting groups. In M. Schaller, J. Simpson, \& D Kenrick (Eds.), Evolution and social psychology (pp. 143-161). New York, NY: Psychology Press.

Brewer, M. B., \& Kramer, R. M. (1986). Choice behavior in social dilemmas: Effects of social identity, group size, and decision framing. Journal of Personality and Social Psychology, 50, 543-549. doi: 10.1037/0022-3514.50.3.543

Butt, A. N., \& Choi, J. N. (2010). Does power matter? Negotiator status as a moderator of the relationship between negotiator emotion and behav- 
ior. International Journal of Conflict Management, 21, 124-146. doi: 10.1108/10444061011037378

*Caldwell, M. D. (1976). Communication and sex effects in a five-person prisoner's dilemma game. Journal of Personality and Social Psychology, 33, 273-280. doi:10.1037/0022-3514.33.3.273

*Camera, G., \& Casari, M. (2009). Cooperation among strangers under the shadow of the future. American Economic Review, 99, 979-1005. doi: 10.1257/aer.99.3.979

Caporael, L. R. (1997). The evolution of truly social cognition: The core configurations model. Personality and Social Psychology Review, 1, 276-298. doi:10.1207/s15327957pspr0104_1

Cardenas, J. C., Stranlund, J., \& Willis, C. (2002). Economic inequality and burden-sharing in the provision local environmental quality. Ecological Economics, 40, 379-395. doi:10.1016/S0921-8009(01)00285-3

Carpenter, J. P. (2007a). The demand for punishment. Journal of Economic Behavior \& Organization, 62, 522-542. doi:10.1016/j.jebo.2005.05.004

*Carpenter, J. P. (2007b). Punishing free-riders: How group size affects mutual monitoring and the provision of public goods. Games and Economic Behavior, 60, 31-51. doi:10.1016/j.geb.2006.08.011

Carpenter, J., Bowles, S., Gintis, H., \& Hwang, S. (2009). Strong reciprocity and team production. Journal of Economic Behavior \& Organization, 71, 221-232. doi:10.1016/j.jebo.2009.03.011

*Carpenter, J., \& Matthews, P. H. (2009). What norms trigger punishment? Experimental Economics, 12, 272-288. doi:10.1007/s10683-009-9214-z

*Carpenter, J. P., Matthews, P., \& Ong'ong'a, O. (2004). Why punish? Social reciprocity and the enforcement of prosocial norms. Journal of Evolutionary Economics, 14, 407-429. doi:10.1007/s00191-004-0212-1

Casari, M. (2005). On the design of peer punishment experiments. Experimental Economics, 8, 107-115. doi:10.1007/s10683-005-0869-9

*Casari, M., \& Luini, L. (2009). Cooperation under alternative punishment institutions: An experiment. Journal of Economic Behavior \& Organization, 71, 273-282. doi:10.1016/j.jebo.2009.03.022

*Cha, Y. S. (2000). Using the concepts of framing and loss aversion to answer pertinent questions on cooperative behaviors in social dilemmas. Dissertation Abstracts International: Section B. Sciences and Engineering, 60(5), 5831

Chaudhuri, A. (2011). Sustaining cooperation in laboratory public goods experiments: A selective survey of the literature. Experimental Economics, 14, 47-83. doi:10.1007/s10683-010-9257-1

*Chen, X., Pillutla, M. M., \& Yao, X. (2009). Unintended consequences of cooperation inducing and maintaining mechanisms in public goods dilemmas: Sanctions and moral appeals. Group Processes \& Intergroup Relations, 12, 241-255. doi:10.1177/1368430208098783

*Cinyabuguma, M., Page, T., \& Putterman, L. (2005). Cooperation under the threat of expulsion in a public goods experiment. Journal of Public Economics, 89, 1421-1435. doi:10.1016/j.jpubeco.2004.05.011

*Clark, J. (2002). Recognizing large donations to public goods: An experimental test. Managerial and Decision Economics, 23, 33-44. doi: $10.1002 /$ mde. 1044

Cooper, H. M. (1998). Synthesizing research: A guide for literature reviews (3rd ed.). Thousand Oaks, CA: Sage.

Dawes, R. M. (1980). Social dilemmas. Annual Review of Psychology, 31, 169-193. doi:10.1146/annurev.ps.31.020180.001125

Deci, E. L., Koestner, R., \& Ryan, R. M. (1999). A meta-analytic review of experiments examining the effects of extrinsic rewards on intrinsic motivation. Psychological Bulletin, 125, 627-668. doi:10.1037/00332909.125.6.627

Deci, E. L., \& Ryan, R. M. (2000). The "what" and "why" of goal pursuits: Human needs and the self-determination of behavior. Psychological Inquiry, 11, 227-268. doi:10.1207/S15327965PLI1104_01

De Cremer, D., \& van Dijk, E. (2005). When and why leaders put themselves first: Leader behaviour in resource allocations as a function of feeling entitled. European Journal of Social Psychology, 35, 553563. doi:10.1002/ejsp. 260
De Cremer, D., \& van Dijk, E. (2008). Leader-follower effects in resource dilemmas: The roles of leadership selection and social responsibility. Group Processes \& Intergroup Relations, 11, 355-369. doi:10.1177/ 1368430208090647

De Dreu, C. K. W., \& van Kleef, G. A. (2004). The influence of power on the information search, impression formation, and demands in negotiation. Journal of Experimental Social Psychology, 40, 303-319. doi: 10.1016/j.jesp.2003.07.004

Denant-Boemont, L., Masclet, D., \& Noussair, C. N. (2007). Punishment, counterpunishment, and sanction enforcement in a social dilemma experiment. Economic Theory, 33, 145-167. doi:10.1007/s00199-0070212-0

*Dickinson, D. L. (2001). The carrot vs. the stick in work team motivation. Experimental Economics, 4, 107-124. doi:10.1007/BF01669275

*Dreber, A., Rand, D. G., Fudenberg, D., \& Nowak, M. A. (2008, March 20). Winners don't punish. Nature, 452, 348-351. doi:10.1038/ nature 06723

Driscoll, M. P. (2005). Psychology of learning for instruction. Boston, MA: Peterson.

Duval, S., \& Tweedie, R. (2000). Trim and fill: A simple funnel-plot-based method of testing and adjusting for publication bias in meta-analysis. Biometrics, 56, 455-463. doi:10.1111/j.0006-341X.2000.00455.x

Edney, J. J., \& Harper, C. S. (1978). The commons dilemma: A review of contributions in psychology. Environmental Management, 2, 491-507. doi:10.1007/BF01866708

*Eek, D., Loukopoulos, P., Satoshi, F., \& Gärling, T. (2002). Spill-over effect of intermittent costs for defection in social dilemmas. European Journal of Social Psychology, 32, 801-813. doi:10.1002/ejsp.122

*Egas, M., \& Riedl, A. (2008). The economics of altruistic punishment and the maintenance of cooperation. Proceedings of the Royal Society: Series B. Biological Sciences, 275, 871-878. doi:10.1098/ rspb.2007.1558

*Ertan, A., Page, T., \& Putterman, L. (2009). Who to punish? Individual decisions and majority rule in mitigating the free rider problem. European Economic Review, 53, 495-511. doi:10.1016/j.euroecorey 2008.09.007

Falk, A., Fehr, E., \& Fischbacher, U. (2005). Driving forces of informal sanctions. Econometrica, 73, 2017-2030. doi:10.1111/j.14680262.2005.00644.x

Fehr, E., \& Fischbacher, U. (2004). Social norms and human cooperation. Trends in Cognitive Sciences, 8, 185-190. doi:10.1016/j.tics .2004 .02 .007

Fehr, E., Fischbacher, U., \& Gächter, S. (2002). Strong reciprocity, human cooperation, and the enforcement of social norms. Human Nature, 13, 1-25. doi:10.1007/s12110-002-1012-7

*Fehr, E., \& Gächter, S. (2000). Cooperation and punishment in public goods experiments. American Economic Review, 90, 980-994. doi: 10.1257/aer.90.4.980

*Fehr, E., \& Gächter, S. (2002, January 10). Altruistic punishment in humans. Nature, 415, 137-140. doi:10.1038/415137a

Fehr, E., \& Schmidt, K. M. (1999). A theory of fairness, competition, and cooperation. Quarterly Journal of Economics, 114, 817-868. doi: $10.1162 / 003355399556151$

Fowler, J. H., Johnson, T., \& Smirnov, O. (2005, January 6). Egalitarian motives and altruistic punishment. Nature, 433, E1. doi:10.1038/ nature 03256

French, J. R. P., Jr., \& Raven, B. (1959). The bases of social power. In D. Cartwright (Ed.), Studies in social power (pp. 150-167). Ann Arbor: University of Michigan, Institute for Social Research.

*Fuster, A., \& Meier, S. (2010). Another hidden cost of incentives: The detrimental effect on norm enforcement. Management Science, 56, $57-$ 70. doi: $10.1287 / \mathrm{mnsc} .1090 .1081$

*Gächter, S., \& Herrmann, B. (2009). Reciprocity, culture and human cooperation: Previous insights and a new cross-cultural experiment. 
Philosophical Transactions of the Royal Society: Series B. Biological Sciences, 364, 791-806. doi:10.1098/rstb.2008.0275

*Gächter, S., \& Herrmann, B. (2011). The limits of self-governance when cooperators get punished: Experimental evidence from urban and rural Russia. European Economic Review, 55, 193-210. doi:10.1016/ j.euroecorev.2010.04.003

Gächter, S., Herrmann, B., \& Thöni, C. (2010). Culture and cooperation. Philosophical Transactions of the Royal Society: Series B. Biological Sciences, 365, 2651-2661. doi:10.1098/rstb.2010.0135

*Gächter, S., Renner, E., \& Sefton, M. (2008, December 5). The long-run benefits of punishment. Science, 322, 1510. doi:10.1126/science .1164744

*Gächter, S., \& Thöni, C. (2005). Social learning and voluntary cooperation among like minded people. Journal of European Economic Association, 3, 303-314. doi:10.1162/jeea.2005.3.2-3.303

Gershoff, E. T. (2002). Corporate punishment by parents and associated child behaviors and experiences: A meta-analytic and theoretical review. Psychological Bulletin, 128, 539-579. doi:10.1037/0033-2909.128 .4 .539

Gintis, H. (2008, March 7). Punishment and cooperation. Science, 319, 1345-1346. doi:10.1126/science. 1155333

Gneezy, U., \& Rustichini, A. (2000). A fine is a price. Journal of Legal Studies, 29, 1-17. doi:10.1086/468061

Gürerk, Ö., Irlenbusch, B., \& Rockenbach, B. (2006, April 7). The competitive advantage of sanctioning institutions. Science, 312, 108-111. doi:10.1126/science. 1123633

Hardin, G. (1968, December 13). The tragedy of the commons. Science, 162, 1243-1248. doi:10.1126/science.162.3859.1243

Hedges, L. V., \& Olkin, I. (1985). Statistical methods for meta-analysis. Orlando, FL: Academic Press.

Henrich, J., Boyd, R., Bowles, S., Camerer, C., Fehr, E., Gintis, H., \& McElreath, R. (2001). In search of homo economicus: Behavioral experiments in 15 small-scale societies. American Economic Review, 91, 73-78. doi:10.1257/aer.91.2.73

*Herrmann, B., Thöni, C., \& Gächter, S. (2008, March 7). Antisocial punishment across societies. Science, 319, 1362-1367. doi:10.1126/ science. 1153808

Hobbes, T. (1651). Leviathan. Cambridge, England: Cambridge University Press.

Hogg, M. A., \& Terry, D. J. (2000). Social identity and self-categorization processes in organizational contexts. Academy of Management Review, 25, 121-140. doi:10.2307/259266

Holz, W. C., \& Azrin, N. H. (1962). Interactions between the discriminative and aversive properties of punishment. Journal of the Experimental Analysis of Behavior, 5, 229-234. doi:10.1901/jeab.1962.5-229

*Hopfensitz, A., \& Rueben, E. (2009). The importance of emotions for the effectiveness of social punishment. Economic Journal, 119, 1534-1559. doi:10.1111/j.1468-0297.2009.02288.x

Inglehart, R., \& Baker, W. E. (2000). Modernization, cultural change, and the persistence of traditional values. American Sociological Review, 65, 19-51. doi:10.2307/2657288

Kalkhoff, W., \& Barnum, C. (2000). The effects of status-organizing and social identity processes on patterns of social influence. Social Psychology Quarterly, 63, 95-115. doi:10.2307/2695886

Kazdin, A. E. (2001). Behavior modification in applied settings. Belmont, CA: Wadsworth

Kelley, H. H., Holmes, J. G., Kerr, N. L., Reis, H. T., Rusbult, C. E., \& Van Lange, P. A. M. (2003). An atlas of interpersonal situations. Cambridge, England: Cambridge University Press.

Kelley, H. H., \& Thibaut, J. W. (1978). Interpersonal relations: A theory of interdependence. New York, NY: Wiley.

Keltner, D., Langner, C. A., \& Allison, M. L. (2006). Power and moral leadership. In D. Rhode (Ed.). Moral leadership: The theory and prac- tice of power, judgment, and policy (pp. 177-194). San Francisco, CA Jossey-Bass.

Keltner, D., van Kleef, G. A., Chen, S., \& Kraus, M. W. (2008). A reciprocal influence model of social power: Emerging principles and lines of inquiry. Advances in Experimental Social Psychology, 40, 151192. doi:10.1016/S0065-2601(07)00003-2

Kerr, N. L. (1989). Illusions of efficacy: The effects of group size on perceived efficacy in social dilemmas. Journal of Experimental Social Psychology, 25, 287-313. doi:10.1016/0022-1031(89)90024-3

Kerr, N. L., Rumble, A. C., Park, E., Ouwerkerk, J. W., Parks, C. D., Gallucci, M., \& Van Lange, P. A. M. (2009). How many bad apples does it take to spoil the whole barrel? Social exclusion and toleration for bad apples. Journal of Experimental Social Psychology, 45, 603-613. doi: 10.1016/j.jesp.2009.02.017

*Kieruj, N., Mulder, L. B., \& Nelissen, R. M. A. (2008). Opdrijven of beklijven? Het verschil tussen boetes en sociale afkeuring in sociale dilemma's [Forcing up or sinking in? The difference between fines and social disapproval in social dilemmas]. Jaarboek Sociale Psychologie, 2007, 193-202. doi:urn:nbn:nl:ui:12-3132860

*Kocher, M. G., Martinsoon, P., \& Visser, M. (2008). Does stake size matter for cooperation and punishment? Economic Letters, 99, 508-511. doi:10.1016/j.econlet.2007.09.048

Kollock, P. (1998). Social dilemmas: The anatomy of cooperation. Annual Review of Sociology, 24, 183-214. doi:10.1146/annurev.soc.24.1.183

*Komorita, S. S., \& Barth, J. M. (1985). Components of reward in social dilemmas. Journal of Personality and Social Psychology, 48, 364-373. doi:10.1037/0022-3514.48.2.364

Komorita, S. S., \& Parks, C. D. (1995). Social dilemmas. Boulder, CO Westview Press.

Komorita, S. S., Sheposh, J. P., \& Braver, S. L. (1968). Power, the use of power, and cooperative choice in a two-person game. Journal of Personality and Social Psychology, 8, 134-142. doi:10.1037/h0025300

Kramer, R. M. (1999). Trust and distrust in organizations: Emerging perspectives, enduring questions. Annual Review of Psychology, 50, 569-598. doi:10.1146/annurev.psych.50.1.569

*Kroll, S., Cherry, T. L., \& Shogren, J. F. (2007). Voting, punishment, and public goods. Economic Inquiry, 45, 557-570. doi:10.1111/j.14657295.2007.00028.x

Lepper, M. R., \& Greene, M. D. (1978). The hidden costs of reward. Hillsdale, NJ: Erlbaum.

Lipsey, M. W., \& Wilson, D. B. (2001). Practical meta-analysis. London, England: Sage.

Lynn, M., \& Oldenquist, A. (1986). Egoistic and nonegoistic motives in social dilemmas. American Psychologist, 41, 529-534. doi:10.1037/ 0003-066X.41.5.529

*Martichuski, D. K., \& Bell, P. A. (1991). Reward, punishment, privatization, and moral suasion in a commons dilemma. Journal of Applied Social Psychology, 21, 1356-1369. doi:10.1111/j.1559-1816.1991 .tb00475.x

Masclet, D., Noussair, C., Tucker, S., \& Villeval, M. (2003). Monetary and nonmonetary punishment in the voluntary contributions mechanism. American Economic Review, 93, 366-380. doi:10.1257/ 000282803321455359

*McCusker, C., \& Carnevale, P. J. (1995). Framing in resource dilemmas Loss aversion and the moderating effects of sanctions. Organizational Behavior and Human Decision Processes, 61, 190-201. doi:10.1006/ obhd.1995.1015

Messick, D. M., Wilke, H. A. M., Brewer, M. B., Kramer, R. M., Zemke, P. E., \& Lui, L. (1983). Individual adaptations and structural change as solutions to social dilemmas. Journal of Personality and Social Psychology, 44, 294-309. doi:10.1037/0022-3514.44.2.294

*Mulder, L. B. (2005). [Sanctions and social values in a public good dilemma]. Unpublished raw data, Department of Human Resource Man- 
agement and Organizational Behavior, University of Groningen, Groningen, the Netherlands.

*Mulder, L. B. (2008). The difference between punishments and rewards in fostering moral concerns in social decision making. Journal of Experimental Social Psychology, 44, 1436-1443. doi:10.1016/j.jesp .2008 .06 .004

Mulder, L. B., \& Nelissen, R. M. A. (2011). When rules really make a difference: The effect of cooperation rules on moral norms in social dilemmas. Journal of Business Ethics. Advance online publication. doi:10.1007/s10551-011-0795-z

*Mulder, L. B., van Dijk, E., De Cremer, D., \& Wilke, H. A. M. (2001). [Sanctions, rewards and trust in a public good dilemma]. Unpublished raw data, Department of Human Resource Management and Organizational Behavior, University of Groningen, Groningen, the Netherlands.

*Mulder, L. B., van Dijk, E., De Cremer, D., \& Wilke, H. A. M. (2002). [Sanctions and trust in a public good dilemma]. Unpublished raw data, Department of Human Resource Management and Organizational Behavior, University of Groningen, Groningen, the Netherlands.

*Mulder, L. B., van Dijk, E., De Cremer, D., \& Wilke, H. A. M. (2003). [Sanctions and trust in a public good dilemma]. Unpublished raw data, Department of Human Resource Management and Organizational Behavior, University of Groningen, Groningen, the Netherlands.

*Mulder, L. B., van Dijk, E., De Cremer, D., \& Wilke, H. A. M. (2006a). Undermining trust and cooperation: The paradox of sanctioning systems in social dilemmas. Journal of Experimental Social Psychology, 42, 147-162. doi:10.1016/j.jesp.2005.03.002

*Mulder, L. B., van Dijk, E., De Cremer, D., \& Wilke, H. A. M. (2006b). When sanctions fail to increase cooperation in social dilemmas: Considering the presence of an alternative defection option. Personality and Social Psychology Bulletin, 32, 1312-1324. doi:10.1177/ 0146167206289978

Mulder, L. B., van Dijk, E., Wilke, H. A. M., \& De Cremer, D. (2005). The effect of feedback on support for a sanctioning system in a social dilemma: The difference between installing and maintaining the sanction. Journal of Economic Psychology, 26, 443-458. doi:10.1016/ j.joep.2004.12.007

*Mulder, L. B., \& van Prooijen, J. W. (2006). [Sanctions and framing in social dilemmas]. Unpublished raw data, Department of Human Resource Management and Organizational Behavior, University of Groningen, Groningen, the Netherlands.

Mulder, L. B., Verboon, P., \& De Cremer, D. (2009). Sanctions and moral judgments: The moderating effect of sanction severity and trust in authorities. European Journal of Social Psychology, 39, 255-269. doi: 10.1002/ejsp.506

Murray, S. L., \& Holmes, J. G. (2009). The architecture of interdependent minds: A motivation-management theory of mutual responsiveness. Psychological Review, 116, 908-928. doi:10.1037/a0017015

Muthusamy, S. K., \& White, M. A. (2006). Does power sharing matter? The role of power and influence in alliance performance. Journal of Business Research, 59, 811-819. doi:10.1016/j.jbusres.2006.01.018

*Myers, C. D. (2009). Participation and punishment. Unpublished manuscript, Department of Politics, Princeton University.

*Nelissen, R. M. A., \& Mulder, L. B. (2010). What makes a sanction "stick"? The effects of financial and social sanctions on norm compliance. Unpublished manuscript, Department of Social Psychology, Tilburg University, Tilburg, the Netherlands.

*Nikiforakis, N. (2008). Punishment and counter-punishment in public good games: Can we really govern ourselves? Journal of Public Economics, 92, 91-112. doi:10.1016/j.jpubeco.2007.04.008

*Nikiforakis, N., \& Normann, H. (2008). A comparative statistical analysis of punishment in public-good experiments. Experimental Economics, 11, 358-369. doi:10.1007/s10683-007-9171-3

*Nikiforakis, N., Normann, H., \& Wallace, B. (2010). Asymmetric en- forcement of cooperation in a social dilemma. Southern Economic Journal, 76, 638-659. doi:10.4284/sej.2010.76.3.638

Noussair, C., \& Tucker, S. (2005). Combining monetary and social sanctions to promote cooperation. Economic Inquiry, 43, 649-660. doi: 10.1093/ei/cbi045

Nowak, M. A., \& Sigmund, K. (2005, October 27). Evolution of indirect reciprocity. Nature, 437, 1291-1298. doi:10.1038/nature04131

*O'Gorman, R., Henrich, J., \& Van Vugt, M. (2008). Constraining free riding in public goods games: Designated solitary punishers can sustain human cooperation. Proceedings of the Royal Society: Series B. Biology, 276, 323-329. doi:10.1098/rspb.2008.1082

*O'Gorman, R., \& Van Vugt, M. (2010). [Does allowing participants the option to choose their punisher increase contributions in a public goods game.] Unpublished raw data, Department of Psychology, University of Essex, Colchester, England.

Oldmeadow, J. A., Platow, M. J., Foddy, M., \& Anderson, D. (2003). Self-categorization, status, and social influence. Social Psychology Quarterly, 66, 138-152. doi:10.2307/1519844

Oliver, P. (1980). Rewards and punishments as selective incentives for collective action: Theoretical investigations. American Journal of Sociology, 85, 1356-1375. doi:10.1086/227168

Olson, M. (1965). The logic of collective action: Public goods and the theory of groups. Cambridge, MA: Harvard University Press.

Orwin, R. G. (1983). A fail-safe $N$ for effect size in meta-analysis. Journal of Educational Statistics, 8, 157-159. doi:10.2307/1164923

Ostrom, E., Walker, J., \& Gardner, R. (1992). Covenants with and without the sword: Self-governance is possible. American Political Science Review, 86, 404-417. doi:10.2307/1964229

Overbeck, J. R., Neale, M. A., \& Govan, C. L. (2010). I feel, therefore you act: Intrapersonal and interpersonal effects of emotion on negotiation as a function of social power. Organizational Behavior and Human Decision Processes, 112, 126-139. doi:10.1016/j.obhdp.2010.02.004

*Page, T., Putterman, L., \& Unel, B. (2005). Voluntary association in public goods experiments: Reciprocity, mimicry, and efficiency. Economic Journal, 115, 1032-1053. doi:10.1111/j.1468-0297.2005.01031.x

*Parks, C. D. (2000). Testing various types of cooperation rewards in social dilemma. Group Processes \& Intergroup Relations, 3, 339-350. doi:10.1177/1368430200003004001

Parks, C. D., Sanna, L. J., \& Berel, S. R. (2001). Actions of similar others as inducements to cooperate in social dilemmas. Personality and Social Psychology Bulletin, 27, 345-354. doi:10.1177/0146167201273008

*Patel, A., Cartwright, E., \& Van Vugt, M. (2010). Punishment cannot sustain cooperation in a public good game with free-rider anonymity. Unpublished manuscript, Department of Economics, University of Gothenburg, Gothenburg, Sweden

Pruitt, D. G., \& Kimmel, M. J. (1977). Twenty years of experimental gaming: Critique, synthesis, and suggestions for the future. Annual Review of Psychology, 28, 363-392. doi:10.1146/annurev.ps.28 .020177 .002051

*Rand, D. G., Dreber, A., Ellingsen, T., Fudenberg, D., \& Nowak, M. A. (2009, September 4). Positive interactions promote public cooperation. Science, 325, 1272-1275. doi:10.1126/science.1177418

*Rapoport, A., \& Au, W. T. (2001). Bonus and penalty in common pool resource dilemmas under uncertainty. Organizational Behavior and $\mathrm{Hu}$ man Decision Processes, 85, 135-165. doi:10.1006/obhd.2000.2935

Rapoport, A., \& Chammah, A. M. (1965). Prisoner's dilemma: A study in conflict and cooperation. Ann Arbor: University of Michigan Press.

*Reuben, E., \& Riedl, A. (2009). Enforcement of contribution norms in public goods games with heterogeneous populations. Unpublished manuscript, Management Division, Columbia University, New York, NY.

*Riedl, A., \& Ule, A. (2009). Exclusion and cooperation in social network experiments. Unpublished manuscript, Faculty of Economics and Econometrics, Center for Research in Experimental Economics and political Decision-making (CREED), Amsterdam, the Netherlands. 
Rockenbach, B., \& Milinski, M. (2006, December 7). The efficient interaction of indirect reciprocity and costly punishment. Nature, 444, $718-$ 723. doi:10.1038/nature05229

Rusbult, C. E., \& Van Lange, P. A. M. (2003). Interdependence, interaction, and relationships. Annual Review of Psychology, 54, 351-375. doi:10.1146/annurev.psych.54.101601.145059

Rutte, C. G., \& Wilke, H. A. M. (1984). Social dilemmas and leadership. European Journal of Social Psychology, 14, 105-121. doi:10.1002/ ejsp. 2420140109

Ryan, R. M., \& Deci, E. L. (2000). Self-determination theory and the facilitation of intrinsic motivation, social development, and well-being. American Psychologist, 55, 68-78. doi:10.1037110003-066X.55.1.68

Samuelson, C. D. (1991). Perceived task difficulty, causal attributions, and preferences for structural change in resource dilemmas. Personality and Social Psychology Bulletin, 17, 181-187. doi:10.1177/ 014616729101700210

Samuelson, C. D., \& Allison, S. T. (1994). Cognitive factors affecting the use of social decision heuristics in resource-sharing tasks. Organizational Behavior and Human Decision Processes, 58, 1-27. doi:10.1006/ obhd.1994.1027

*Sato, K. (1987). Distribution of the cost of maintaining common resources. Journal of Experimental Social Psychology, 23, 19-31. doi: 10.1016/0022-1031(87)90023-0

*Sefton, M., Shupp, R., \& Walker, J. M. (2007). The effect of rewards and sanctions in provision of public goods. Economic Inquiry, 45, 671-690. doi:10.1111/j.1465-7295.2007.00051.x

*Sell, J., \& Wilson, R. K. (1999). The maintenance of cooperation: Expectations of future interaction and the trigger of group punishment. Social Forces, 77, 1551-1571. doi:10.2307/3005886

* Shaw, J. I. (1976). Response-contingent payoffs and cooperative behavior in the prisoner's dilemma game. Journal of Personality and Social Psychology, 34, 1024-1033. doi:10.1037/0022-3514.34.5.1024

* Shinada, M., \& Yamagishi, T. (2007). Punishing free riders: Direct and indirect promotion of cooperation. Evolution and Human Behavior, 28, 330-339. doi:10.1016/j.evolhumbehav.2007.04.001

Shinada, M., \& Yamagishi, T. (2008). Bringing back the leviathan into social dilemmas. In A. Biel, D. Eek, T. Garling, \& M. Gukstafsson (Eds.), New issues and paradigms in research on social dilemmas (pp. 93-123). New York, NY: Springer. doi:10.1007/978-0-387-72596-3_7

Sigmund, K. (2007). Punish or perish? Retaliation and collaboration among humans. Trends in Ecology \& Evolution, 22, 593-600. doi:10.1016/ j.tree.2007.06.012

Sinaceur, M., \& Tiedens, L. Z. (2006). Get mad and get more than even: The benefits of anger expressions in negotiations. Journal of Experimental Social Psychology, 42, 314-322. doi:10.1016/j.jesp.2005.05.002

Skinner, B. F. (1953). Science of human behavior. New York, NY: Macmillan.

Stern, P. C. (1976). Effect of incentives and education on resource conservation decisions in a simulated common dilemma. Journal of Personality and Social Psychology, 34, 1285-1292. doi:10.1037/00223514.34.6.1285

* Sutter, M., Haigner, S., \& Kocher, M. G. (2010). Choosing the carrot or the stick? Endogenous institutional choice in social dilemma situations. Review of Economic Studies, 77, 1540-1566. doi:10.1111/j.1467937X.2010.00608 $\mathrm{x}$

*Sutter, M., Linder, P., \& Platsch, D. (2009). Social norms, third-party observation, and third-party rewards. Unpublished manuscript, Department of Public Finance, University of Innsbruck, Innsbruck, Austria.

*Tan, F. (2008). Punishment in a linear public good game with productivity heterogeneity. De Economist, 156, 269-293. doi:10.1007/s10645-0089094-1

*Tenbrunsel, A. E., \& Messick, D. M. (1999). Sanctioning systems, decision frames, and cooperation. Administrative Science Quarterly, 44, 684-707. doi:10.2307/2667052
Thibaut, J. W., \& Kelley, H. H. (1959). The social psychology of groups. New York, NY: Wiley.

Turner, J. C. (1991). Social influence. Milton Keynes, England: Open University Press.

*Tyran, J., \& Feld, L. P. (2004). Achieving compliance when Legal sanctions are non-deterrent. Unpublished manuscript, Department of Economics, University of Copenhagen, Copenhagen, Denmark.

van Dijk, E., \& De Cremer, D. (2006). Self-benefiting in the allocation of scarce resources: Leader-follower effects and the moderating effect of social value orientations. Personality and Social Psychology Bulletin, 32, 1352-1361. doi:10.1177/0146167206290338

van Kleef, G. A., De Dreu, C. K. W., \& Manstead, A. S. R. (2004). The interpersonal effects of anger and happiness in negotiations. Journal of Personality and Social Psychology, 86, 57-76. doi:10.1037/00223514.86.1.57

Van Lange, P. A. M. (1999). The pursuit of joint outcomes and equality in outcomes: An integrative model of social value orientation. Journal of Personality and Social Psychology, 77, 337-349. doi:10.1037/00223514.77.2.337

Van Lange, P. A. M., De Cremer, D., van Dijk, E., \& Van Vugt, M. (2007). Self-interest and beyond: Basic principles of social interaction. In A. W. Kruglanski \& E. T. Higgins (Eds.), Social psychology: Handbook of basic principles (pp. 540-561). New York, NY: Guilford Press.

Van Lange, P. A. M., \& Joireman, J. A. (2008). How we can promote behavior that serves all of us in the future. Social Issues and Policy Review, 2, 127-157. doi:10.1111/j.1751-2409.2008.00013.x

Van Lange, P. A. M., Ouwerkerk, J. W., \& Tazelaar, M. J. A. (2002). How to overcome the detrimental effects of noise in social interaction: The benefits of generosity. Journal of Personality and Social Psychology, 82, 768-780. doi:10.1037/0022-3514.82.5.768

Van Lange, P. A. M., \& Rusbult, C. E. (in press). Interdependence theory. In P. A. M. Van Lange, A. W. Kruglanski, \& E. T. Higgins. (Eds.), Handbook of theories of social psychology. Thousand Oaks, CA: Sage.

Van Lange, P. A. M., Schippers, M. C., \& Balliet, D. (in press). Who volunteers in psychology experiments? An empirical review of prosocial motivation in volunteering. Personality and Individual Differences

*van Prooijen, J., Gallucci, M., \& Toeset, G. (2008). Procedural justice in punishment systems: Inconsistent punishment procedures have detrimental effects on cooperation. British Journal of Social Psychology, 47, 311-324. doi:10.1348/014466607X218212

*Van Vugt, M., \& De Cremer, D. (1999). Leadership in social dilemmas: The effects of group identification on collective actions to provide public goods. Journal of Personality and Social Psychology, 76, 587-599. doi:10.1037/0022-3514.76.4.587

Van Vugt, M., Hogan, R., \& Kaiser, R. B. (2008). Leadership, followership, and evolution: Some lessons from the past. American Psychologist, 63, 182-196. doi:10.1037/0003-066X.63.3.182

*Walker, J. M., \& Halloran, M. A. (2004). Rewards and sanctions and the provision of public goods in one-shot settings. Experimental Economics, 7. 235-247. doi:10.1023/B:EXEC.0000040559.08652.51

Wieselquist, J., Rusbult, C. E., Foster, C. A., \& Agnew, C. R. (1999) Commitment, pro-relationship behavior, and trust in close relationships. Journal of Personality and Social Psychology, 77, 942-966. doi: 10.1037/0022-3514.77.5.942

*Wit, A., \& Wilke, H. A. (1990). The presentation of rewards and punishments in a simulated social dilemma. Social Behaviour, 5, 231-245.

*Xiao, E., \& Kunreuther, H. (2010). Punishment and cooperation in stochastic social dilemmas. Unpublished manuscript, The Wharton School, University of Pennsylvania.

*Yamagishi, T. (1986). The provisioning of a sanctioning system as a public good. Journal of Personality and Social Psychology, 51, 110116. doi:10.1037/0022-3514.51.1.110

*Yamagishi, T. (1988). Seriousness of social dilemmas and the provision 
of a sanctioning system. Social Psychology Quarterly, 51, 32-42. doi: $10.2307 / 2786982$

*Yamagishi, T. (1992). Group size and the provision of a sanctioning system in a social dilemma. In W. B. G. Liebrand, D. M. Messick, \& H. A. M. Wilke (Eds.), Social dilemma: Theoretical issues and research findings (pp. 267-287). Oxford, England: Pergamon Press.

Zelmer, J. (2003). Linear public goods experiments: A meta-analysis. Experimental Economics, 6, 299-310. doi:10.1023/A:1026277420119
Zimring, F. (1971). Perspectives on deterrence. Washington, DC: National Institute of Mental Health.

Received May 23, 2010

Revision received February 16, 2011

Accepted February 22, 2011

\section{Online First Publication}

APA-published journal articles are now available Online First in the PsycARTICLES database. Electronic versions of journal articles will be accessible prior to the print publication, expediting access to the latest peer-reviewed research.

All PsycARTICLES institutional customers, individual APA PsycNET ${ }^{\circledR}$ database package subscribers, and individual journal subscribers may now search these records as an added benefit. Online First Publication (OFP) records can be released within as little as 30 days of acceptance and transfer into production, and are marked to indicate the posting status, allowing researchers to quickly and easily discover the latest literature. OFP articles will be the version of record; the articles have gone through the full production cycle except for assignment to an issue and pagination. After a journal issue's print publication, OFP records will be replaced with the final published article to reflect the final status and bibliographic information. 